\title{
Cu-Catalyzed Asymmetric Allylic Alkylations of Aromatic and Aliphatic Phosphates with Alkylzinc Reagents. An Effective Method for Enantioselective Synthesis of Tertiary and Quaternary Carbons
}

\author{
Monica A. Kacprzynski and Amir H. Hoveyda* \\ Department of Chemistry, Merkert Chemistry Center, Boston College \\ Chestnut Hill, Massachusetts 02467
}

\section{Supporting Information}

General. Infrared (IR) Spectra are recorded on a Nicolet 210 spectrophotometer, $v_{\max }$ in $\mathrm{cm}^{-1}$. Bands are characterized as broad (br), strong (s), medium (m), or weak (w). ${ }^{1} \mathrm{H}$ NMR spectra are recorded on a Varian Unity INOVA $400(400 \mathrm{MHz})$ spectrometer. Chemical shifts are reported in ppm from tetramethylsilane with the solvent resonance resulting from incomplete deuteration as the internal standard $\left(\mathrm{CDCl}_{3}: \delta 7.26\right)$. Data are reported as follows: chemical shift, integration, multiplicity $(\mathrm{s}=$ singlet, $\mathrm{d}=$ doublet, $\mathrm{t}=$ triplet, $\mathrm{q}=$ quartet, $\mathrm{br}=$ broad and $\mathrm{m}=$ multiplet), coupling constants, and assignment. For products $\mathbf{5 a}-\mathbf{5 i}$, where a mixture of $\mathrm{S}_{\mathrm{N}} 2$ ' and $\mathrm{S}_{\mathrm{N}} 2$ exists, data is presented only for the $\mathrm{S}_{\mathrm{N}} 2$ ' product in the ${ }^{1} \mathrm{H}$ NMR. ${ }^{13} \mathrm{C}$ NMR spectra are recorded on a Varian Unity INOVA $400(100 \mathrm{MHz})$ spectrometer with complete proton decoupling. Chemical shifts are reported in ppm from tetramethylsilane with the solvent resonance as the internal standard $\left(\mathrm{CDCl}_{3}: \delta 77.16\right)$. For products $\mathbf{5 d}, \mathbf{5 h}$, and $\mathbf{5 i}$, data is presented for the $\mathrm{S}_{\mathrm{N}} 2$ ' and $\mathrm{S}_{\mathrm{N}} 2$ product mixture in the ${ }^{13} \mathrm{C}$ NMR. Enantiomer ratios are determined by chiral GLC analysis; (Alltech Associates Chiraldex GTA column (30 m x $0.25 \mathrm{~mm}$ )), Alphadex 120 column $(30 \mathrm{~m} \times 0.25 \mathrm{~mm})$, or Betadex 120 column $(30 \mathrm{~m} \times 0.25 \mathrm{~mm})$ or by analytical liquid chromatography (HPLC) on a Shimadzu chromatograph with a Chiralpak AS $(4.6 \times 250 \mathrm{~mm})$ or a Chiralcel OJ $(4.6 \times 250 \mathrm{~mm})$ chiral column by Chiral Technologies in comparison with authentic racemic materials. $\mathrm{S}_{\mathrm{N}} 2^{\prime}: \mathrm{S}_{\mathrm{N}} 2$ ratios are determined by achiral GLC analysis (J\&W Scientific HP-5, $30 \mathrm{~m} \times 0.25 \mathrm{~mm}$ ) or by 400 $\mathrm{MHz}{ }^{1} \mathrm{H}$ NMR. Elemental analyses are performed by Robertson Microlit Laboratories (Madison, New Jersey). High resolution mass spectrometry is performed by the University of Illinois Mass Spectrometry Laboratories (Urbana, Illinois) and by Mr. Chris Kowalczyk (on a Micromass LCT ESI-MS (positive mode)) at the Mass Spectrometry Facility, Boston College. Optical Rotation values are recorded on a Rudolph Research Analytical Autopol IV Polarimeter.

Unless otherwise stated, all reactions are conducted in oven $\left(135^{\circ} \mathrm{C}\right)$ and flamedried glassware under an inert atmosphere of nitrogen. THF, $\mathrm{Et}_{2} \mathrm{O}$, and $\mathrm{CH}_{2} \mathrm{Cl}_{2}$ are purified through two alumina columns under a positive pressure of dry argon by a modified Advanced ChemTech purification system. Diethylzinc and dimethylzinc are purchased from Strem and used without purification. Other alkylzinc reagents are prepared according to known methods. ${ }^{1}$ Copper (I) triflate (benzene complex, 2:1) is

\footnotetext{
${ }^{1}$ P. Knochel, R. Singer, Chem. Rev. 1993, 93, 2177-2188 and references cited therein
} 
prepared using known methods, ${ }^{2}$ while copper (I) triflate (toluene complex, 2:1) is purchased from Aldrich and used without purification. Copper (I) cyanide is purchased from Strem chemicals and used without further purification. The allylic alcohols were prepared from the corresponding aldehydes and ketones by a two step Horner-Emmons olefination $/ \mathrm{LiAlH}_{4}$ reduction sequence, ${ }^{3}$ with the exception of compounds containing nitro groups and disubstituted $p-\mathrm{CF}_{3}$ group, which were reduced using DIBAL-H. Allylic alcohols were converted to allylic phosphates using known procedures. ${ }^{3} \mathrm{EDC} \cdot \mathrm{HCl}$, $\mathrm{HOBt} \cdot \mathrm{H}_{2} \mathrm{O}$, DIC, piperidine, butylamine, DMF, Boc- and Fmoc-protected amino acids, and all salicyl aldehydes are purchased from commercial sources and used without further purification. Triethylamine is distilled from $\mathrm{CaH}_{2}$. Gly-Wang resin $(0.6 \mathrm{mmol} / \mathrm{g})$ is purchased from Advanced ChemTech.

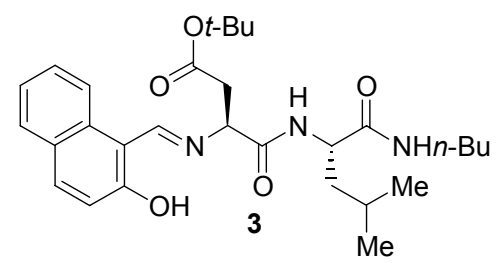

2-Hydroxy-1-napthaldehyde- L-Asp (Ot-Bu)-L-LeuNHn-Bu: Boc-L-Leu-OH (1.00 g, $4.0 \mathrm{mmol})$ is dissolved in $10 \mathrm{~mL} \mathrm{CH}_{2} \mathrm{Cl}_{2}$ and $\mathrm{HOBt} \cdot \mathrm{H}_{2} \mathrm{O}(0.61 \mathrm{~g}, 4.0 \mathrm{mmol})$ and $\mathrm{EDC} \cdot \mathrm{HCl}(0.76 \mathrm{~g}, 4.0 \mathrm{mmol})$ are added successively. The solution is cooled to $0{ }^{\circ} \mathrm{C}$ with an ice bath and $n$ butylamine $(0.79 \mathrm{~mL}, 8.0 \mathrm{mmol})$ is added. The mixture is allowed to warm to room temperature and stir for $3 \mathrm{~h}$. Reaction is quenched by addition of $20 \mathrm{~mL} 10 \mathrm{wt} . \%$ aqueous citric acid, and the solution is diluted by addition of $20 \mathrm{~mL}$ $\mathrm{CH}_{2} \mathrm{Cl}_{2}$. The organic layer is washed with $20 \mathrm{~mL}$ of a saturated aqueous solution of $\mathrm{NaHCO}_{3}$ and $20 \mathrm{~mL}$ of a saturated aqueous solution of $\mathrm{NaCl}$, dried over anhydrous $\mathrm{MgSO}_{4}$, filtered and concentrated to yield a white solid.

Trifluoroacetic acid $(2 \mathrm{~mL})$ is added dropwise to Boc-L-Leu-NHn-Bu at $0{ }^{\circ} \mathrm{C}$ and allowed to stir for 30 minutes at which time the reaction is quenched by the dropwise addition of $2 \mathrm{~N}$ aqueous solution of $\mathrm{NaOH}$ until the $\mathrm{pH}$ is $>10$. The aqueous solution is then washed with $\mathrm{CH}_{2} \mathrm{Cl}_{2}(3 \times 10 \mathrm{~mL})$. The organic layer is washed with $10 \mathrm{~mL}$ of a saturated aqueous solution of $\mathrm{NaCl}$, dried over anhydrous $\mathrm{MgSO}_{4}$, filtered and concentrated to afford $\mathrm{H}_{2} \mathrm{~N}-\mathrm{L}-\mathrm{Leu}-\mathrm{NH} n-\mathrm{Bu}(0.52 \mathrm{~g}, 2.8 \mathrm{mmol}, 70 \%)$ as a yellow oil.

To a solution of $\mathrm{H}_{2} \mathrm{~N}-\mathrm{L}-\mathrm{Leu}-\mathrm{NH} n-\mathrm{Bu}(0.52 \mathrm{~g}, 2.8 \mathrm{mmol})$ and Fmoc-L-Asp (Ot$\mathrm{Bu})-\mathrm{OH}(1.2 \mathrm{~g}, 2.8 \mathrm{mmol})$ in $7 \mathrm{~mL} \mathrm{CH} \mathrm{Cl}_{2}, \mathrm{HOBt} \cdot \mathrm{H}_{2} \mathrm{O}(0.43 \mathrm{~g}, 2.8 \mathrm{mmol})$ and $\mathrm{EDC} \cdot \mathrm{HCl}$ $(0.53 \mathrm{~g}, 2.8 \mathrm{mmol})$ are added and the reaction is cooled to $0{ }^{\circ} \mathrm{C}$ with an ice bath. $\mathrm{Et}_{3} \mathrm{~N}$ $(0.40 \mathrm{~mL}, 2.8 \mathrm{mmol})$ is added and the solution is allowed to warm to room temperature. The solution is allowed to stir for $12 \mathrm{~h}$ at which time the reaction is quenched by addition of $20 \mathrm{~mL} 10 \mathrm{wt} . \%$ aqueous citric acid and the resulting solution is diluted by addition of $20 \mathrm{~mL} \mathrm{CH} \mathrm{CH}_{2}$. The organic layer is washed with $20 \mathrm{~mL}$ of a saturated aqueous solution of $\mathrm{NaHCO}_{3}$ and $20 \mathrm{~mL}$ of a saturated aqueous solution of $\mathrm{NaCl}$, dried over anhydrous $\mathrm{MgSO}_{4}$, filtered and concentrated to yield a white solid. Fmoc-L-Asp $(\mathrm{O} t \mathrm{Bu})-\mathrm{L}-\mathrm{LeuNH} n$ $\mathrm{Bu}$ is dissolved in $10 \mathrm{~mL} \mathrm{CH} \mathrm{Cl}_{2}$ and the flask is cooled to $0{ }^{\circ} \mathrm{C}$. Piperidine $(1.39 \mathrm{~mL}, 14$ mmol) is added and the solids slowly dissolve. The mixture is allowed to stir at $0{ }^{\circ} \mathrm{C}$ for 30 minutes at which time the resulting solution is concentrated. The mixture is purified

\footnotetext{
2 (a) R. G. Salomon, J. K. Kochi, J. Am. Chem. Soc. 1973, 95, 1889-1897. (b) R. G. Salomon, J. K. Kochi, J. Am Chem. Soc. 1973, 95, 3300-3310.

${ }^{3}$ C. A. Luchaco-Cullis, H. Mizutani, K. E. Murphy, A. H. Hoveyda, Angew. Chem. Int. Ed. 2001, 40, 14561460.
} 
by silica gel chromatography (EtOAc to 10:1 EtOAc:MeOH) to yield the desired amine (0.73 g, $2.1 \mathrm{mmol}, 75 \%)$.

$\mathrm{H}_{2} \mathrm{~N}-\mathrm{L}-\mathrm{Asp}(\mathrm{O} t \mathrm{Bu})-\mathrm{L}-\mathrm{LeuNH} n$-Bu $(0.79 \mathrm{~g}, 2.2 \mathrm{mmol})$ is dissolved in $5.5 \mathrm{~mL}$ $\mathrm{CH}_{2} \mathrm{Cl}_{2}$ and 2-hydroxy-1-napthaldehyde $(0.38 \mathrm{~g}, 2.2 \mathrm{mmol})$ is added followed by addition of anhydrous $\mathrm{MgSO}_{4}(0.64 \mathrm{~g}, 4.4 \mathrm{mmol})$. The mixture is allowed to stir at room temperature for $12 \mathrm{~h}$. At which time anhydrous $\mathrm{MgSO}_{4}$ is filtered and the solution is concentrated to yield a light brown solid. The solid is purified by silica gel chromatography $\left(1: 1\right.$ hexanes:EtOAc, $\mathrm{R}_{f}$ product $\left.=0.45\right)$ to yield a bright yellow solid (0.78 g, $1.5 \mathrm{mmol}, 68 \%$ ). mp 143-145 ${ }^{\circ} \mathrm{C}$. IR (solid film): 3270 (br), 3081 (m), 2955 (m), 2917 (m), $2861(\mathrm{w}), 1728$ (s), $1646(\mathrm{~s}), 1539(\mathrm{~m}), 1375(\mathrm{~m}), 1155(\mathrm{~m}), 834(\mathrm{w}), 746$ (m) $\mathrm{cm}^{-1} .{ }^{1} \mathrm{H}$ NMR $\left(400 \mathrm{MHz}, \mathrm{CDCl}_{3}\right): \delta 9.27(1 \mathrm{H}, \mathrm{s}, \mathbf{H C}=\mathrm{N}), 8.06(1 \mathrm{H}, \mathrm{d}, J=8.4 \mathrm{~Hz}$, $\operatorname{ArH}), 7.83(1 \mathrm{H}, \mathrm{d}, J=8.8 \mathrm{~Hz}, \operatorname{ArH}), 7.75(1 \mathrm{H}, \mathrm{dd}, J=8.0,0.8 \mathrm{~Hz}, \operatorname{ArH}), 7.53(1 \mathrm{H}, \mathrm{ddd}$, $J=7.2,7.2,1.2 \mathrm{~Hz}, \operatorname{ArH}), 7.36(1 \mathrm{H}, \mathrm{ddd}, J=7.6,7.6,1.2 \mathrm{~Hz}, \operatorname{ArH}), 7.14(1 \mathrm{H}, \mathrm{d}, J=8.8$ $\mathrm{Hz}, \operatorname{ArH}), 6.53(1 \mathrm{H}, \mathrm{d}, J=7.6 \mathrm{~Hz}, \mathrm{NH}), 6.16(1 \mathrm{H}, \mathrm{br}, \mathrm{NH}), 4.45-4.37(2 \mathrm{H}, \mathrm{m}$, $\mathrm{NCHCO}), 3.26-3.15\left(2 \mathrm{H}, \mathrm{m}, \mathrm{NCH}_{2}\right), 3.13$ (1H, dd, $\left.J=16.4,4.4 \mathrm{~Hz}, \mathrm{CHCHHCO}\right), 2.81$ $(1 \mathrm{H}, \mathrm{dd}, J=16.4,8.8 \mathrm{~Hz}, \mathrm{CHCHHCO}), 1.77-1.54(3 \mathrm{H}, \mathrm{m}$, aliphatic $\mathrm{CH}), 1.49-1.40(2 \mathrm{H}$, $\mathrm{m}$, aliphatic CH), $1.36(9 \mathrm{H}, \mathrm{s}, t-\mathbf{B u}), 1.35-1.26(2 \mathrm{H}, \mathrm{m}$, aliphatic $\mathbf{C H}), 0.94(3 \mathrm{H}, \mathrm{d}, J=$ $\left.6.4 \mathrm{~Hz}, \mathrm{CH}\left(\mathrm{CH}_{3}\right)_{2}\right), 0.93\left(3 \mathrm{H}, \mathrm{d}, J=6.4 \mathrm{~Hz}, \mathrm{CH}\left(\mathrm{CH}_{3}\right)_{2}\right), 0.88(3 \mathrm{H}, \mathrm{dd}, J=7.2,7.2 \mathrm{~Hz}$, $\left.\mathrm{CH}_{3}\right) .{ }^{13} \mathrm{C}$ NMR $\left(100 \mathrm{MHz}, \mathrm{CDCl}_{3}\right): \delta 171.5,170.7,170.1,165.3,165.0,136.1,133.1$, 129.6, 128.4, 128.0, 124.0, 120.6, 119.0, 108.9, 82.0, 68.7, 52.5, 41.0, 40.1, 39.7, 31.8, 28.4, 25.3, 23.2, 22.5, 20.4, 14.0. HRMS Calcd for $\mathrm{C}_{29} \mathrm{H}_{41} \mathrm{~N}_{3} \mathrm{O}_{5} \mathrm{Na}\left(\mathrm{M}+\mathrm{Na}^{+}\right)$: 534.2942; Found: 534.2944. $[\alpha]_{\mathrm{D}}^{20}+3.5^{\circ}\left(c=0.87, \mathrm{CHCl}_{3}\right)$.

Representative procedure for Cu-catalyzed allylic substitution of dialkylzinc reagents with unsymmetrical allylic phosphates. (CAUTION: $\mathrm{Et}_{2} \mathrm{Zn}$ is pyrophoric! Use extreme caution!) In the glove box, a flask is charged with $\left(40.2 \mathrm{mg}, 8.00 \times 10^{-1}\right.$ mmol $)(\mathrm{CuOTf})_{2} \cdot \mathrm{PhH}$ and $(81.8 \mathrm{mg}, 1.60 \mathrm{mmol})$ 2-hydroxy-1-napthaldehyde-L-Asp $(\mathrm{O} t$ $\mathrm{Bu})-\mathrm{L}-\mathrm{LeuNH} n-\mathrm{Bu}(3)$, sealed with a septum, and removed from the glovebox. Upon addition of $10.0 \mathrm{~mL}$ THF into the flask, the mixture is cooled to $-15{ }^{\circ} \mathrm{C}$ in a dry ice/acetone bath and allowed to stir for $5 \mathrm{~min}$ before the addition of $(500 \mu \mathrm{L}, 4.80 \mathrm{mmol})$ $\mathrm{Et}_{2} \mathrm{Zn}$. A solution of 1-(2-nitrophenyl)-3-(diethylphosphoryloxy)-1-propene (504 mg, $1.60 \mathrm{mmol}$ ) in $10.0 \mathrm{~mL}$ THF is then added. The mixture is allowed to stir for 24 hours at $-15{ }^{\circ} \mathrm{C}$, at which time the reaction is quenched with the addition of $10 \mathrm{~mL}$ of a saturated aqueous $\mathrm{NH}_{4} \mathrm{Cl}$ solution. The resulting solution is washed three times with $10 \mathrm{~mL} \mathrm{Et}_{2} \mathrm{O}$ and the combined organic layers are dried over anhydrous $\mathrm{MgSO}_{4}$. The volatiles are removed in vacuo (CAUTION: Product is volatile!) to yield a yellow oil. The oil is purified by silica gel chromatography $\left(100 \%\right.$ pentane to $50: 1$ pentane: $\mathrm{Et}_{2} \mathrm{O}$ to elute product) to yield the desired product as a light yellow oil (290 mg, $1.52 \mathrm{mmol}, 95 \%)$.

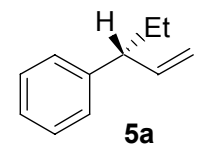

(R)-(-)-3-Phenyl-1-pentene (9:1 mixture of $S_{\mathbf{N}} \mathbf{2}$, to $S_{\mathbf{N}} \mathbf{2}$ products). IR (neat): 3087 (w), $3069(\mathrm{~m}), 2961$ (s), 2924 (s), $2880(\mathrm{~m}), 1645$ (m), 1608 (m), 1495 (s), 1464 (s), 1382 (w), 916 (s), 696 (s) $\mathrm{cm}^{-1} .{ }^{1} \mathrm{H}$ NMR (400 $\left.\mathrm{MHz}, \mathrm{CDCl}_{3}\right): \delta$ 7.36-7.18 (5H, m, ArH), $5.90(1 \mathrm{H}, \mathrm{ddd}, J=17.2,9.6,7.2 \mathrm{~Hz}$, $\left.\mathrm{CHCH}=\mathrm{CH}_{2}\right), 5.06-5.00\left(2 \mathrm{H}, \mathrm{m}, \mathrm{CHCH}=\mathrm{CH}_{2}\right), 3.14(1 \mathrm{H}, \mathrm{ddd}, J=7.2,7.2,7.2 \mathrm{~Hz}$, $\left.\mathrm{CHCH}=\mathrm{CH}_{2}\right), \quad 1.82-1.67\left(2 \mathrm{H}, \mathrm{m}, \mathrm{CHCH}_{2} \mathrm{CH}_{3}\right), 0.88(3 \mathrm{H}, \mathrm{dd}, J=7.2,7.2 \mathrm{~Hz}$, $\left.\mathrm{CHCH}_{2} \mathrm{CH}_{3}\right) .{ }^{13} \mathrm{C} \mathrm{NMR}\left(100 \mathrm{MHz}, \mathrm{CDCl}_{3}\right): \delta 144.6,142.4,128.5,127.8,126.2,114.2$, 
51.9, 28.5, 12.3. Anal Calcd for $\mathrm{C}_{11} \mathrm{H}_{14}$ : C, 90.35; H, 9.65. Found: C, 89.99; H, 9.39. $[\alpha]_{\mathrm{D}}{ }^{20}-50.6^{\circ}\left(c=0.486, \mathrm{CHCl}_{3}\right)$ for a $95 \%$ ee sample. Proof of Stereochemistry: Literature value $[\alpha]_{\mathrm{D}}{ }^{20}+35.0^{\circ}\left(c=0.6, \mathrm{C}_{6} \mathrm{H}_{6}\right)$ is assigned to the $(S)$ enantiomer. ${ }^{4}$

The optical purity is determined by chiral GLC analysis (Chiraldex-GTA, $45{ }^{\circ} \mathrm{C}, 15 \mathrm{psi}$ ); chromatograms are illustrated below:

Authentic racemic

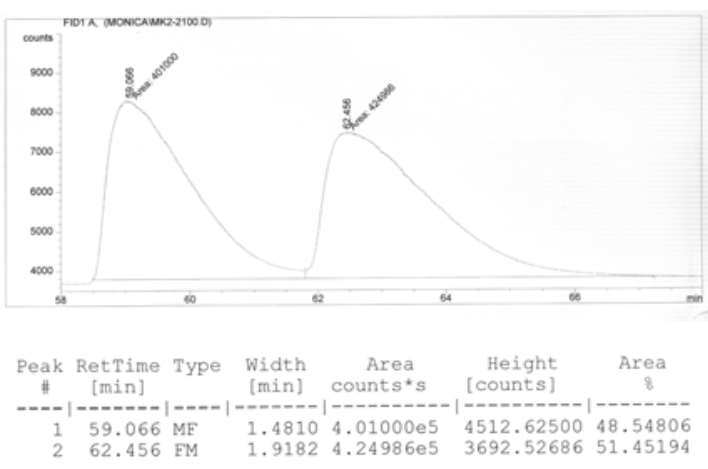

from catalytic reaction

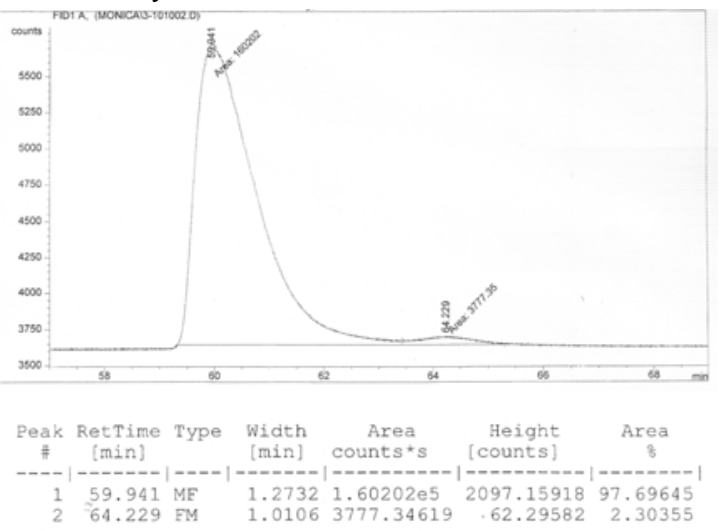

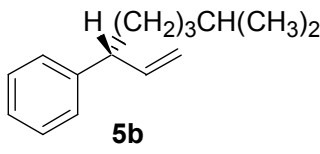

(R)-(-)-7-Methyl-3-phenyl-1-octene (6.7:1 mixture of $S_{N} 2$ ' to $\mathbf{S}_{\mathrm{N}} \mathbf{2}$ products). IR (neat): $3074(\mathrm{w}), 3059(\mathrm{w}), 3024(\mathrm{~m}), 2955(\mathrm{~s})$, $2930(\mathrm{~s}), 2880(\mathrm{~s}), 1640(\mathrm{w}), 1596(\mathrm{w}), 1495(\mathrm{~m}), 1470(\mathrm{w}), 1357$ (w), $1004(\mathrm{w}), 910(\mathrm{~m}) \mathrm{cm}^{-1} .{ }^{1} \mathrm{H}$ NMR $\left(400 \mathrm{MHz}, \mathrm{CDCl}_{3}\right): \delta$ 7.37-7.18 (5H, m, ArH), $5.96\left(1 \mathrm{H}\right.$, ddd, $\left.J=16.4,10.8,7.6 \mathrm{~Hz}, \mathrm{CHCH}=\mathrm{CH}_{2}\right), 5.06-5.01\left(2 \mathrm{H}, \mathrm{m}, \mathrm{CHCH}=\mathrm{CH}_{2}\right)$, $3.25\left(1 \mathrm{H}\right.$, ddd, $\left.J=7.6,7.6,7.6 \mathrm{~Hz}, \mathrm{CHCH}=\mathrm{CH}_{2}\right), 1.74-1.15(7 \mathrm{H}, \mathrm{m}$, aliphatic $\mathrm{CH}), 0.86$ $\left(3 \mathrm{H}, \mathrm{d}, J=6.4 \mathrm{~Hz}, \mathrm{CH}\left(\mathrm{CH}_{3}\right)_{2}\right), 0.85\left(3 \mathrm{H}, \mathrm{d}, J=6.4 \mathrm{~Hz}, \mathrm{CH}\left(\mathrm{CH}_{3}\right)_{2}\right) .{ }^{13} \mathrm{C}$ NMR $(100$ $\left.\mathrm{MHz}, \mathrm{CDCl}_{3}\right): \delta 144.9,142.7,128.5,127.7,126.2,114.0,50.1,39.0,35.8,28.0,25.4$, 22.8, 22.7. HRMS Calc for $\mathrm{C}_{15} \mathrm{H}_{22}:$ 202.1721. Found: 202.1717. $[\alpha]_{\mathrm{D}}{ }^{20}-28.7^{\circ}(c=$ $0.800, \mathrm{CHCl}_{3}$ ) for a $91 \%$ ee sample.

The optical purity is determined by chiral GLC analysis (Chiraldex-GTA, $60{ }^{\circ} \mathrm{C}, 15 \mathrm{psi}$, $t_{\text {minor }}=269$ min, $t_{\text {major }}=271 \mathrm{~min}$ ).

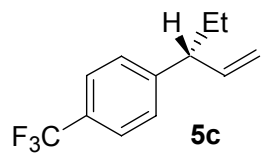

(R)-(-)-3-(4-trifluoromethylphenyl)-1-pentene (9:1 mixture of $\mathbf{S}_{\mathbf{N}}$ 2' to $S_{\mathrm{N}} 2$ products). IR (neat): $2962(\mathrm{~m}), 2923(\mathrm{~m}), 2873(\mathrm{~m}), 1621(\mathrm{~m})$, 1332 (s), 1168 (s), 1130 (s), 1073 (s), 916 (m), $841(\mathrm{~m}) \mathrm{cm}^{-1} .{ }^{1} \mathrm{H}$ NMR $\left(400 \mathrm{MHz}, \mathrm{CDCl}_{3}\right): \delta 7.53(2 \mathrm{H}, \mathrm{d}, J=8.4 \mathrm{~Hz}, \mathrm{ArH}), 7.30(2 \mathrm{H}, \mathrm{d}, J=$ $8.4 \mathrm{~Hz}, \mathrm{ArH}), 5.90\left(1 \mathrm{H}, \mathrm{ddd}, J=17.2,10.4,7.6 \mathrm{~Hz}, \mathrm{CHCH}=\mathrm{CH}_{2}\right), 5.09-5.02(2 \mathrm{H}, \mathrm{m}$, $\left.\mathrm{CHCH}=\mathrm{CH}_{2}\right), 3.21\left(1 \mathrm{H}\right.$, ddd, $\left.J=7.6,7.6,7.6 \mathrm{~Hz}, \mathrm{CHCH}=\mathrm{CH}_{2}\right), 1.84-1.67(2 \mathrm{H}, \mathrm{m}$, $\left.\mathrm{CHCH}_{2} \mathrm{CH}_{3}\right), 0.87\left(3 \mathrm{H}, \mathrm{dd}, J=7.2,7.2 \mathrm{~Hz}, \mathrm{CHCH}_{2} \mathrm{CH}_{3}\right) .{ }^{13} \mathrm{C} \mathrm{NMR}\left(100 \mathrm{MHz}, \mathrm{CDCl}_{3}\right)$ : $\delta$ 148.7, 141.4, 128.5, 128.2, $125.5\left(\mathrm{q}, J=3.4 \mathrm{~Hz}, \mathbf{C F}_{3}\right), 115.1,51.7,28.4,12.2$. HRMS

\footnotetext{
${ }^{4}$ T. Hayashi, T. Hagihara, Y. Katsuro, M. Kumada, Bull. Chem. Soc. Jpn., 1983, 56, 363-364.
} 
Calc for $\mathrm{C}_{12} \mathrm{H}_{13} \mathrm{~F}_{3}$ : 214.0969. Found: 214.0969. $[\alpha]_{\mathrm{D}}^{20}-35.4^{\circ}\left(c=1.03, \mathrm{CHCl}_{3}\right)$ for a $94 \%$ ee sample.

The optical purity is determined by chiral GLC analysis ( $\alpha$-dex, $80{ }^{\circ} \mathrm{C}, 15 \mathrm{psi}$ ); chromatograms are illustrated below:

Authentic racemic

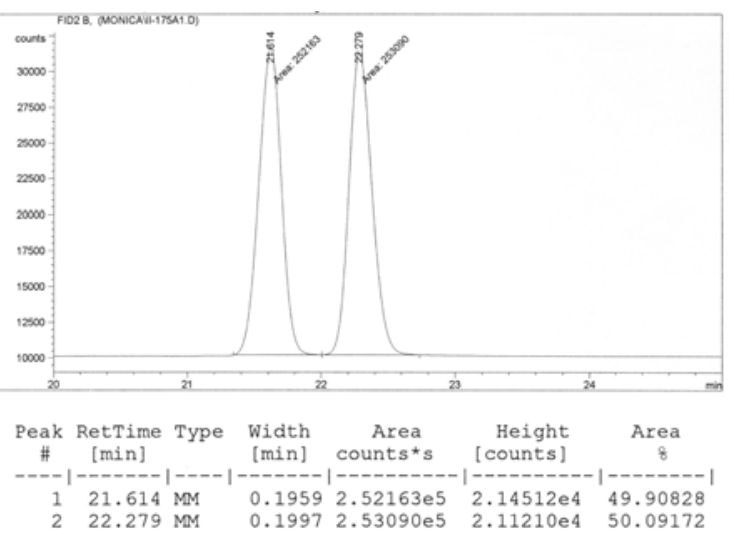

from catalytic reaction

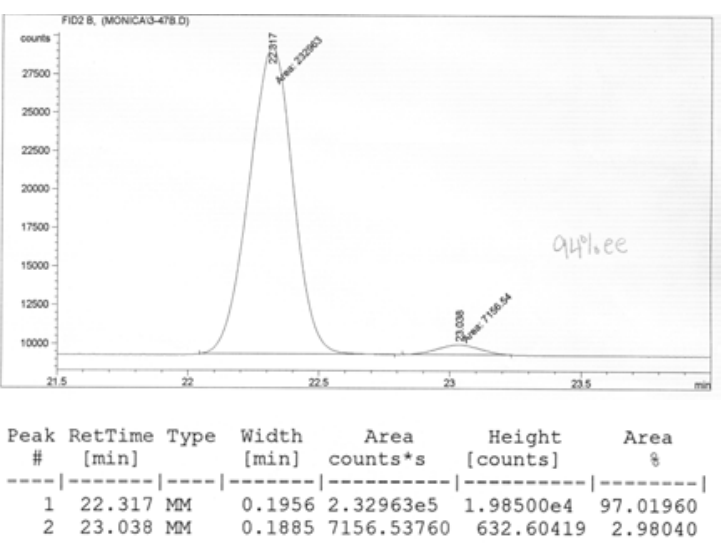

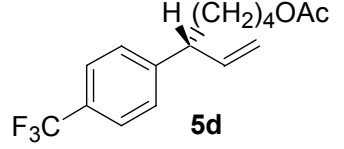

(R)-(-)-7-Acetoxy-3-(4-trifluoromethylphenyl)-1-heptene (4.6:1 mixture of $S_{\mathbf{N}} \mathbf{2}$ ' to $S_{\mathbf{N}} \mathbf{2}$ products). IR (neat): $3075(\mathrm{w}), 2943$ (s), 2861 (s), 1741 (s), 1621 (m), 1331 (s), 1256 (s), 1168 (s), 1123 (s), $1086(\mathrm{~s}), 841(\mathrm{~m}) \mathrm{cm}^{-1} .{ }^{1} \mathrm{H}$ NMR $\left(400 \mathrm{MHz}, \mathrm{CDCl}_{3}\right): \delta 7.56(2 \mathrm{H}, \mathrm{d}$, $J=8.0 \mathrm{~Hz}, \operatorname{ArH}), 7.29(2 \mathrm{H}, \mathrm{d}, J=8.0 \mathrm{~Hz}, \operatorname{ArH}), 5.92(1 \mathrm{H}, \mathrm{ddd}, J=17.6,10.0,7.2 \mathrm{~Hz}$, $\left.\mathrm{CHCH}=\mathrm{CH}_{2}\right), 5.11-5.02\left(2 \mathrm{H}, \mathrm{m}, \mathrm{CHCH}=\mathrm{CH}_{2}\right), 4.03\left(2 \mathrm{H}, \mathrm{dd}, J=6.8,6.8 \mathrm{~Hz}, \mathrm{CH}_{2} \mathrm{OAc}\right)$, $3.31\left(1 \mathrm{H}\right.$, ddd, $\left.J=7.6,7.6,7.6 \mathrm{~Hz}, \mathrm{CHCH}=\mathrm{CH}_{2}\right), 2.02\left(3 \mathrm{H}, \mathrm{s}, \mathrm{CH}_{2} \mathrm{OCOCH}_{3}\right), 1.82-1.21$ $(6 \mathrm{H}, \mathrm{m}$, aliphatic $\mathrm{CH}) \cdot{ }^{13} \mathrm{C}$ NMR $\left(100 \mathrm{MHz}, \mathrm{CDCl}_{3}\right)\left(4.6: 1\right.$ mixture of $\left.\mathrm{S}_{\mathrm{N}} 2^{\prime}: \mathrm{S}_{\mathrm{N}} 2\right): \delta$ 171.3, 171.3, 148.5, 141.2, 133.7, 129.1, 128.8 (q, $\left.J=32.2 \mathrm{~Hz}, \mathrm{ArCCF}_{3}\right), 128.5,126.2$, $125.8,125.6$ (q, $\left.J=3.8 \mathrm{~Hz}, \mathbf{C F}_{3}\right), 123.1,120.4,115.2,64.6,64.4,49.7,34.9,33.0,28.9$, 28.6, 28.6, 25.6, 23.9, 21.1, 21.1. Anal Calcd for $\mathrm{C}_{16} \mathrm{H}_{19} \mathrm{~F}_{3} \mathrm{O}_{2}$ : C, 63.99; H, 6.38. Found: $\mathrm{C}, 63.64 ; \mathrm{H}, 6.14 .[\alpha]_{\mathrm{D}}^{20}-15.2^{\circ}\left(c=1.33, \mathrm{CHCl}_{3}\right)$ for a $84 \%$ ee sample.

The optical purity is determined by chiral GLC analysis ( $\alpha$-dex, $110{ }^{\circ} \mathrm{C}, 15 \mathrm{psi}$ ); chromatograms are illustrated below: 
Authentic racemic
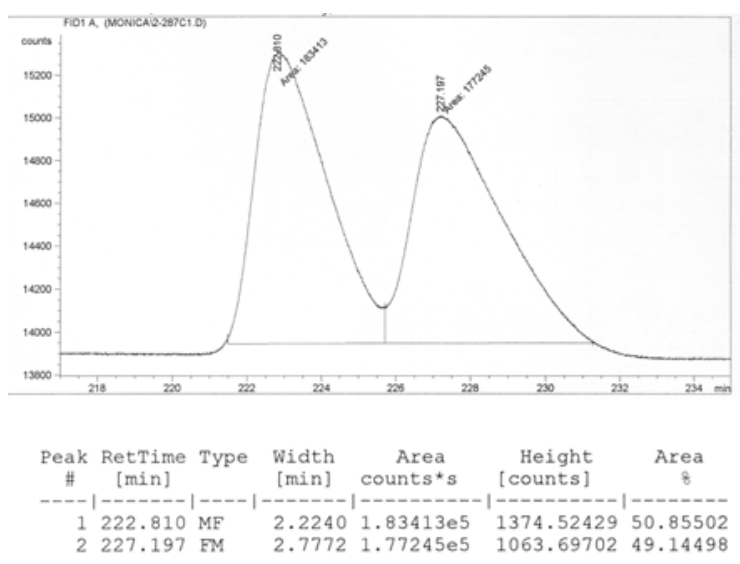

from catalytic reaction

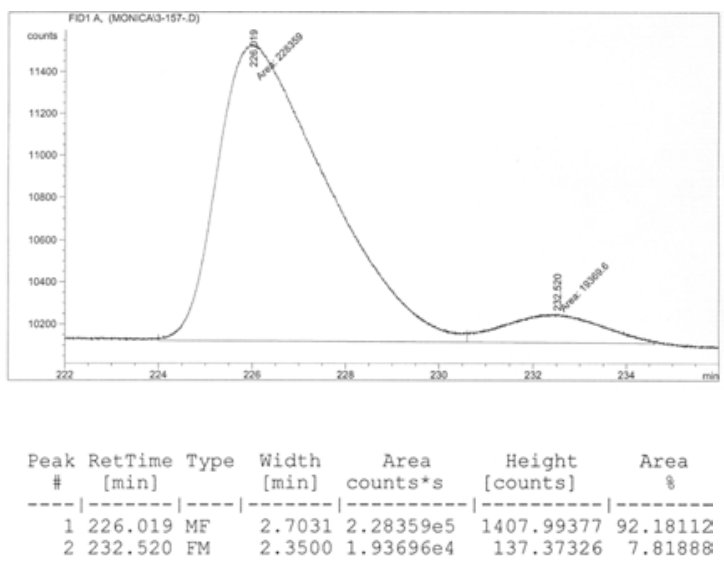

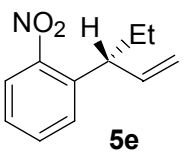

$5 e$

$(R)-(+)-3-(2-N i t r o p h e n y l)-1-p e n t e n e \quad\left(4.9: 1\right.$ mixture of $S_{\mathrm{N}} 2$ ' to $S_{N} 2$ products). IR (neat): 3075 (w), $2967(\mathrm{~s}), 2930(\mathrm{~m}), 2873(\mathrm{~m}), 1596(\mathrm{~m})$, $1608(\mathrm{w}), 1521$ (s), $1357(\mathrm{~s}), 922(\mathrm{~m}), 853(\mathrm{~m}) \mathrm{cm}^{-1}$. ${ }^{1} \mathrm{H}$ NMR (400 MHz, $\left.\mathrm{CDCl}_{3}\right): \delta 7.72(1 \mathrm{H}, \mathrm{dd}, J=8.4,1.2 \mathrm{~Hz}, \operatorname{ArH}), 7.55-7.50(1 \mathrm{H}, \mathrm{m}, \mathrm{ArH})$, $7.40(1 \mathrm{H}, \mathrm{dd}, J=8.0,1.6 \mathrm{~Hz}, \operatorname{ArH}), 7.35-7.30(1 \mathrm{H}, \mathrm{m}, \operatorname{ArH}), 5.92(1 \mathrm{H}, \mathrm{ddd}, J=17.6$, $\left.10.4,7.2 \mathrm{~Hz}, \mathrm{CHCH}=\mathrm{CH}_{2}\right), 5.12-5.05\left(2 \mathrm{H}, \mathrm{m}, \mathrm{CHCH}=\mathrm{CH}_{2}\right), 3.77(1 \mathrm{H}, \mathrm{ddd}, J=7.2,7.2$, $\left.7.2 \mathrm{~Hz}, \mathrm{CHCH}=\mathrm{CH}_{2}\right), 1.85-1.70\left(2 \mathrm{H}, \mathrm{m}, \mathrm{CHCH}_{2} \mathrm{CH}_{3}\right), 0.88(3 \mathrm{H}, \mathrm{dd}, J=7.2,7.2 \mathrm{~Hz}$, $\left.\mathrm{CHCH}_{2} \mathrm{CH}_{3}\right) .{ }^{13} \mathrm{C}$ NMR $\left(100 \mathrm{MHz}, \mathrm{CDCl}_{3}\right): \delta 140.1,132.5,129.1,127.0,124.1,116.0$, 45.0, 28.4, 12.1. Anal Calcd for $\mathrm{C}_{11} \mathrm{H}_{13} \mathrm{NO}_{2}$ : C, 69.09; H, 6.85; N, 7.32. Found: C, $69.07 ; \mathrm{H}, 6.70 ; \mathrm{N}, 7.32 .[\alpha]_{\mathrm{D}}{ }^{20}+4.8^{\circ}\left(c=1.3, \mathrm{CHCl}_{3}\right)$ for a $95 \%$ ee sample.

The optical purity is determined by chiral GLC analysis (Chiraldex-GTA, $130{ }^{\circ} \mathrm{C}, 15$ psi); chromatograms are illustrated below:

Authentic racemic

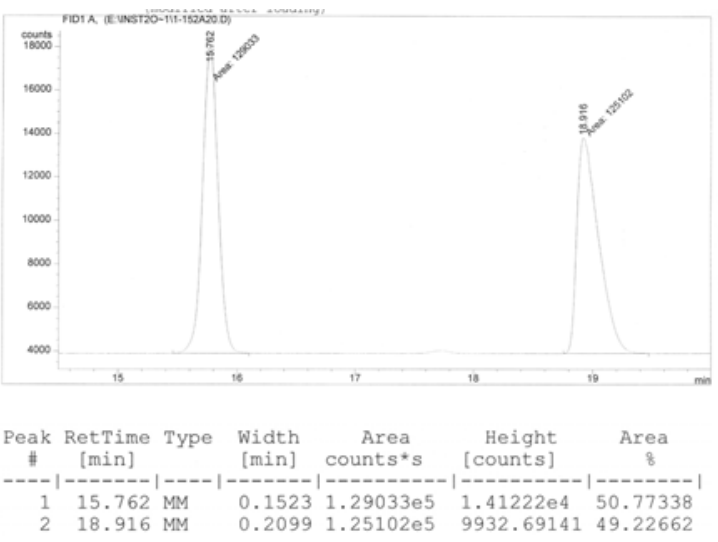

from catalytic reaction

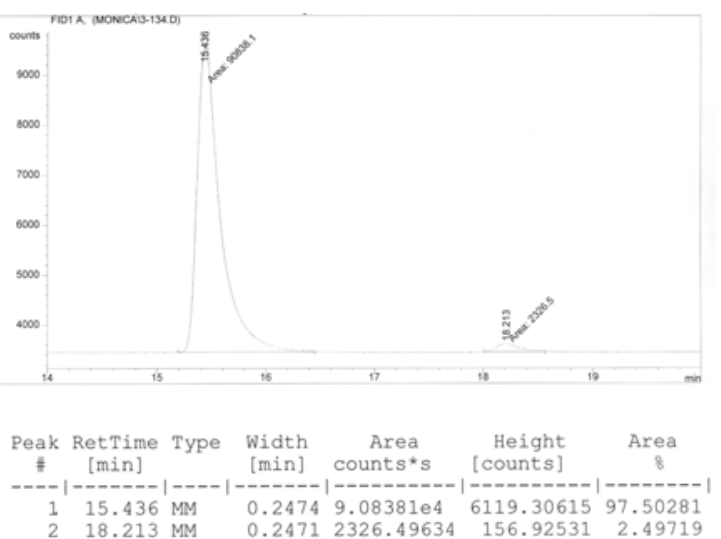

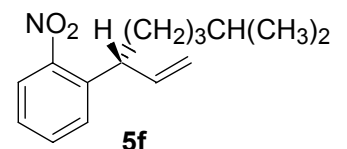

(R)-(-)-7-Methyl-3-(2-nitrophenyl)-1-octene (6.7:1 mixture of $\mathbf{S}_{\mathbf{N}} 2$ ' to $\mathbf{S}_{\mathbf{N}} \mathbf{2}$ products). IR (neat): $3081(\mathrm{w}), 2955$ (s), 2943 (s), $2867(\mathrm{~s}), 1608(\mathrm{~m}), 1527(\mathrm{~s}), 1470(\mathrm{~m}), 1350(\mathrm{~s}), 922(\mathrm{~m}), 784(\mathrm{~m})$, 
$746(\mathrm{~m}) \mathrm{cm}^{-1} .{ }^{1} \mathrm{H}$ NMR $\left(400 \mathrm{MHz}, \mathrm{CDCl}_{3}\right): \delta 7.71(1 \mathrm{H}, \mathrm{dd}, J=8.0,1.2 \mathrm{~Hz}, \mathrm{ArH}), 7.55-$ $7.50(1 \mathrm{H}, \mathrm{m}, \operatorname{ArH}), 7.41(1 \mathrm{H}, \mathrm{dd}, J=8.0,1.2 \mathrm{~Hz}, \operatorname{ArH}), 7.34-7.30(1 \mathrm{H}, \mathrm{m}, \operatorname{ArH}), 5.93$ (1H, ddd, $\left.J=17.6,10.4,7.6 \mathrm{~Hz}, \mathrm{CHCH}=\mathrm{CH}_{2}\right), 5.12-5.04\left(2 \mathrm{H}, \mathrm{m}, \mathrm{CHCH}=\mathrm{CH}_{2}\right), 3.86(1$ $\mathrm{H}$, ddd, $\left.J=7.6,7.6,7.6 \mathrm{~Hz}, \mathrm{CHCH}=\mathrm{CH}_{2}\right), 1.74-1.12(7 \mathrm{H}, \mathrm{m}$, aliphatic $\mathrm{CH}), 0.83(3 \mathrm{H}$, dd, $\left.J=6.4 \mathrm{~Hz}, \mathrm{CH}\left(\mathrm{CH}_{3}\right)_{2}\right), 0.83\left(3 \mathrm{H}, \mathrm{dd}, J=6.4 \mathrm{~Hz}, \mathrm{CH}\left(\mathrm{CH}_{3}\right)_{2}\right) .{ }^{13} \mathrm{C} \mathrm{NMR}(100 \mathrm{MHz}$, $\left.\mathrm{CDCl}_{3}\right): \delta 150.3,140.4,138.8,132.5,129.1,126.9,124.1,115.8,43.3,38.8,35.6,27.9$, 25.2, 22.7, 22.7. Anal Calcd for $\mathrm{C}_{15} \mathrm{H}_{21} \mathrm{NO}_{2}: \mathrm{C}, 72.84 ; \mathrm{H}, 8.56 ; \mathrm{N}, 5.66$. Found: $\mathrm{C}$, $72.61 ; \mathrm{H}, 8.36 ; \mathrm{N}, 5.52 .[\alpha]_{\mathrm{D}}{ }^{20}-10.3^{\circ}\left(c=1.00, \mathrm{CHCl}_{3}\right)$ for a $95 \%$ ee sample.

The optical purity is determined by chiral GLC analysis (Chiraldex-GTA, $140{ }^{\circ} \mathrm{C}, 15$ psi); chromatograms are illustrated below:

Authentic racemic from catalytic reaction
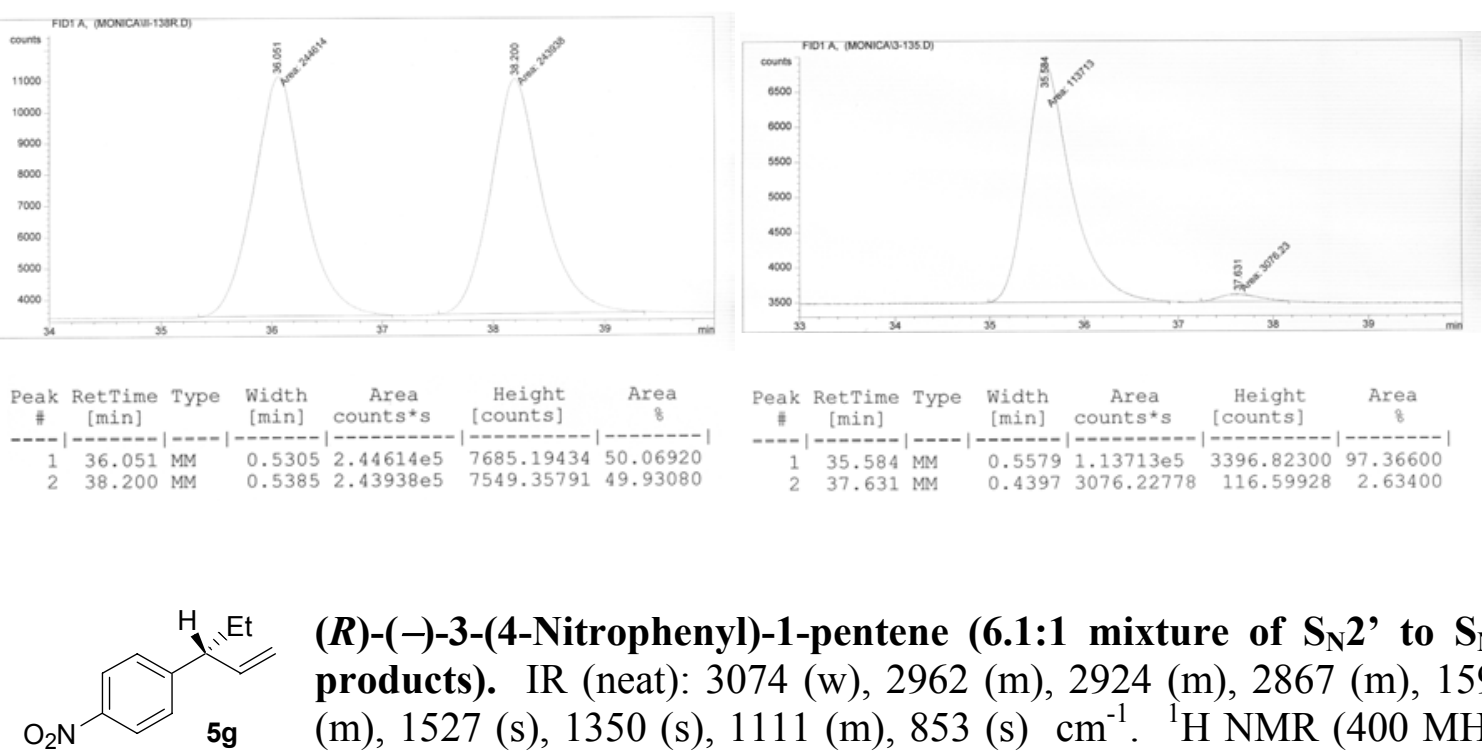

(R)-(-)-3-(4-Nitrophenyl)-1-pentene (6.1:1 mixture of $S_{N} 2$ ' to $S_{N}$ 2 products). IR (neat): $3074(\mathrm{w}), 2962(\mathrm{~m}), 2924(\mathrm{~m}), 2867(\mathrm{~m}), 1596$ (m), $1527(\mathrm{~s}), 1350(\mathrm{~s}), 1111(\mathrm{~m}), 853(\mathrm{~s}) \mathrm{cm}^{-1}$. ${ }^{1} \mathrm{H}$ NMR (400 MHz, $\left.\mathrm{CDCl}_{3}\right): \delta 8.16(2 \mathrm{H}, \mathrm{d}, J=8.8 \mathrm{~Hz}, \mathrm{ArH}), 7.34(2 \mathrm{H}, \mathrm{d}, J=8.8 \mathrm{~Hz}, \mathrm{ArH})$, $5.91\left(1 \mathrm{H}\right.$, ddd, $\left.J=17.2,10.4,7.6 \mathrm{~Hz}, \mathrm{CHCH}=\mathrm{CH}_{2}\right), 5.12-5.04\left(2 \mathrm{H}, \mathrm{m}, \mathrm{CHCH}=\mathrm{CH}_{2}\right)$, $3.27\left(1 \mathrm{H}\right.$, ddd, $\left.J=7.6,7.6,7.6 \mathrm{~Hz}, \mathrm{CHCH}=\mathrm{CH}_{2}\right), 1.86-1.68\left(2 \mathrm{H}, \mathrm{m}, \mathrm{CHCH}_{2} \mathrm{CH}_{3}\right), 0.88$ $\left(3 \mathrm{H}, \mathrm{dd}, J=7.2,7.2 \mathrm{~Hz}, \mathrm{CHCH}_{2} \mathrm{CH}_{3}\right) .{ }^{13} \mathrm{C}$ NMR $\left(100 \mathrm{MHz}, \mathrm{CDCl}_{3}\right): \delta 152.4,140.6$, 128.6, 123.9, 115.7, 51.7, 28.4, 12.1. Anal Calcd for $\mathrm{C}_{11} \mathrm{H}_{13} \mathrm{NO}_{2}$ : C, 69.09; $\mathrm{H}, 6.85 ; \mathrm{N}$, 7.32. Found: $\mathrm{C}, 69.34 ; \mathrm{H}, 6.85 ; \mathrm{N}, 7.36 .[\alpha]_{\mathrm{D}}{ }^{20}-50.3^{\circ}\left(c=0.873, \mathrm{CHCl}_{3}\right)$ for a $94 \%$ ee sample.

The optical purity is determined by chiral GLC analysis ( $\alpha$-dex, $105{ }^{\circ} \mathrm{C}, 15 \mathrm{psi}$ ); chromatograms are illustrated below: 
Authentic racemic
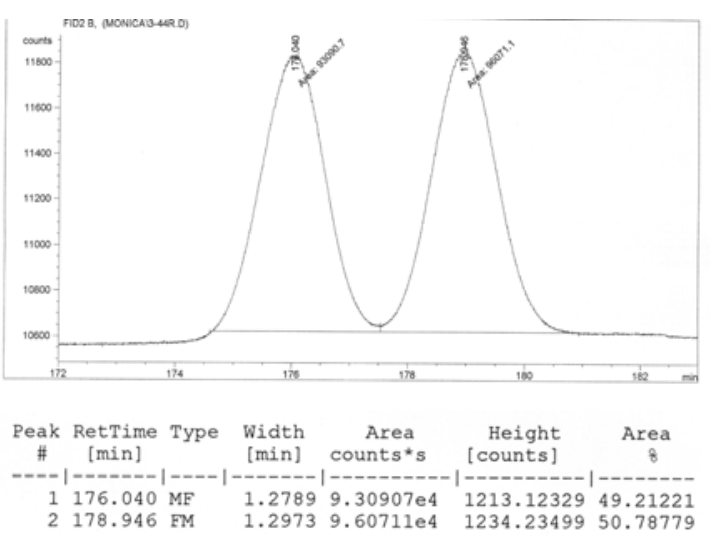

from catalytic reaction

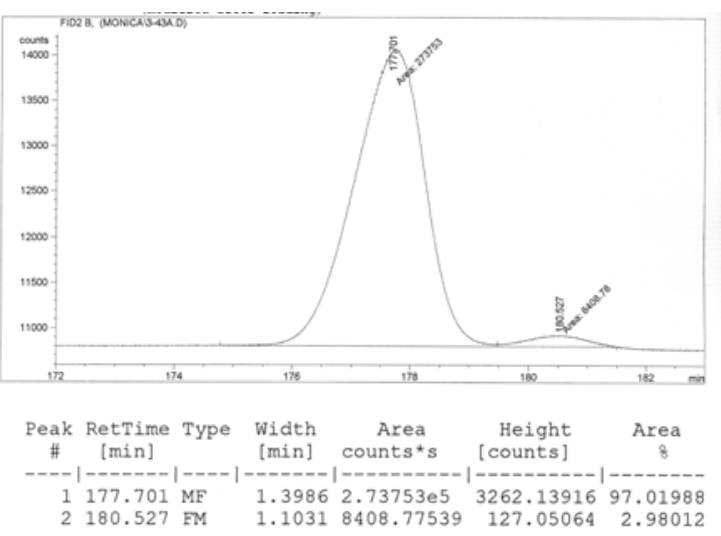

$\underbrace{M e}_{5 h}$

(R)-(-)-3-(2-Methylphenyl)-1-pentene (1:1 mixture of $S_{N} 2$, to $S_{N} 2$ products). IR (neat): 3081 (m), 2962 (s), 2930 (s), 2873 (m), 1495 (m), 1463 (s), 1381 (m), 966 (s), 903 (m), 746 (s) $\mathrm{cm}^{-1} .{ }^{1} \mathrm{H}$ NMR (400 MHz, $\left.\mathrm{CDCl}_{3}\right): \delta 7.19-7.08(4 \mathrm{H}, \mathrm{m}, \mathrm{ArH}), 5.90(1 \mathrm{H}, \mathrm{ddd}, J=17.2,10.4,7.2 \mathrm{~Hz}$, $\left.\mathrm{CHCH}=\mathrm{CH}_{2}\right), 5.04-4.96\left(2 \mathrm{H}, \mathrm{m}, \mathrm{CHCH}=\mathrm{CH}_{2}\right), 3.41(1 \mathrm{H}, \mathrm{ddd}, J=7.6,7.6,7.6 \mathrm{~Hz}$, $\mathrm{CHCH}=\mathrm{CH}_{2}, 2.34(3 \mathrm{H}, \mathrm{s}, \operatorname{ArCH})_{3}, 1.83-1.67\left(2 \mathrm{H}, \mathrm{m}, \mathrm{CHCH}_{2} \mathrm{CH}_{3}\right), 0.91(3 \mathrm{H}, \mathrm{dd}, J=7.2$, $\left.7.2 \mathrm{~Hz}, \mathrm{CHCH}_{2} \mathrm{CH}_{3}\right) .{ }^{13} \mathrm{C} \mathrm{NMR}\left(100 \mathrm{MHz} \mathrm{CDCl}_{3}\right)\left(1: 1\right.$ mixture of $\left.\mathrm{S}_{\mathrm{N}} 2{ }^{\prime}: \mathrm{S}_{\mathrm{N}} 2\right): \delta 142.6$, $141.9,137.2$, 136.1, 135.0, 132.5, 130.4, 130.3, 127.9, 126.9, 126.5, 126.3, 126.1, 125.9, 125.6, 114.1, 46.9, 35.6, 28.0, 22.8, 20.0, 19.8, 13.8, 12.4. HRMS Calcd for $\mathrm{C}_{12} \mathrm{H}_{16}$ : 160.1252. Found: $160.1251 .[\alpha]_{\mathrm{D}}{ }^{20}-15.4^{\mathrm{o}}\left(c=0.933, \mathrm{CHCl}_{3}\right)$ for a $96 \%$ ee sample.

The optical purity is determined by chiral GLC analysis (Chiraldex-GTA, $50{ }^{\circ} \mathrm{C}, 15 \mathrm{psi}$ ); chromatograms are illustrated below:

Authentic racemic
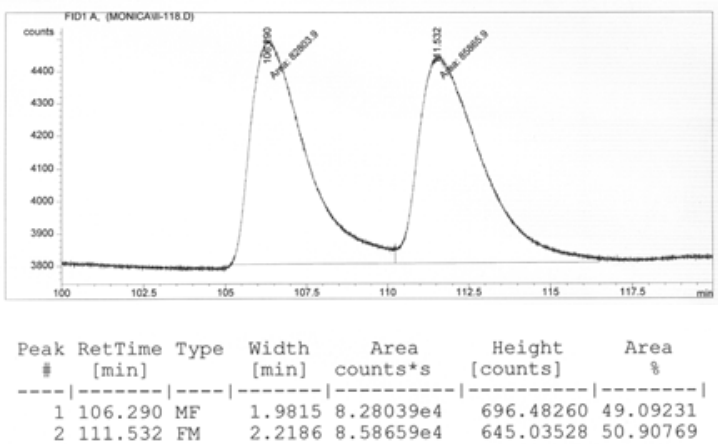

from catalytic reaction

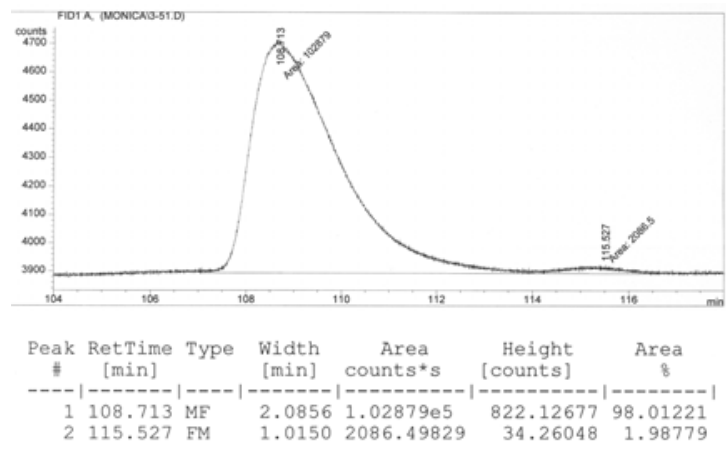

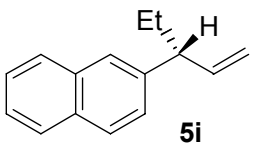

(R)-(-)-3-(2-Napthyl)-1-pentene (1:1.5 mixture of $S_{\mathrm{N}} 2$, to $S_{\mathrm{N}} 2$ products). IR (neat): $3043(\mathrm{~m}), 2962$ (s), 2937 (s), 2880 (m), 1646 (w), $1590(\mathrm{~m}), 1514(\mathrm{~m}), 1451(\mathrm{~m}), 1402(\mathrm{~m}), 973(\mathrm{~s}), 922(\mathrm{~m}), 790(\mathrm{~s}) \mathrm{cm}^{-1}$. ${ }^{1} \mathrm{H}$ NMR $\left(400 \mathrm{MHz}, \mathrm{CDCl}_{3}\right): \delta 8.14(1 \mathrm{H}, \mathrm{d}, J=7.6 \mathrm{~Hz}, \operatorname{ArH}), 7.87-7.83(1 \mathrm{H}, \mathrm{m}, \operatorname{ArH})$, 
7.76-7.72 (1H, m, ArH), 7.53-7.38 (4H, m, ArH), $6.08(1 \mathrm{H}, \mathrm{ddd}, J=17.2,9.6,7.2 \mathrm{~Hz}$, $\left.\mathrm{CHCH}=\mathrm{CH}_{2}\right), 5.13-5.08\left(2 \mathrm{H}, \mathrm{m}, \mathrm{CHCH}=\mathrm{CH}_{2}\right), 4.02(1 \mathrm{H}, \mathrm{ddd}, J=7.2,7.2,7.2 \mathrm{~Hz}$, $\left.\mathrm{CHCH}=\mathrm{CH}_{2}\right), 1.93\left(2 \mathrm{H}, \mathrm{dqd}, J=7.6,7.6,1.6 \mathrm{~Hz}, \mathrm{CHCH}_{2} \mathrm{CH}_{3}\right), 0.97(3 \mathrm{H}, \mathrm{dd}, J=7.6,7.6$ $\left.\mathrm{Hz}, \mathrm{CHCH}_{2} \mathrm{CH}_{3}\right) .{ }^{13} \mathrm{C}$ NMR $\left(100 \mathrm{MHz}, \mathrm{CDCl}_{3}\right)\left(1: 1.5\right.$ mixture of $\left.\mathrm{S}_{\mathrm{N}} 2^{\prime}: \mathrm{S}_{\mathrm{N}} 2\right): \delta 141.9$, 140.6, 136.0, 134.5, 133.8, 131.3, 129.1, 128.6, 127.3, 127.2, 126.8, 125.9, 125.8, 125.7, 125.6, 125.4, 124.1, 123.7, 123.6, 114.8, 46.2, 35.7, 28.2, 22.7, 13.9, 12.5. HRMS Calcd for $\mathrm{C}_{15} \mathrm{H}_{16}$ : 196.1252; Found: 196.1252 . $[\alpha]_{\mathrm{D}}^{20}+6.7^{\circ}\left(c=0.60, \mathrm{CHCl}_{3}\right)$ for a $93 \%$ ee sample.

The optical purity is determined by chiral HPLC analysis (Chiral Technologies Chiralcel OJ, $254 \mathrm{~nm}, 100 \%$ hexanes, $1 \mathrm{~mL} / \mathrm{min}$ ); chromatograms are illustrated below:

Authentic racemic

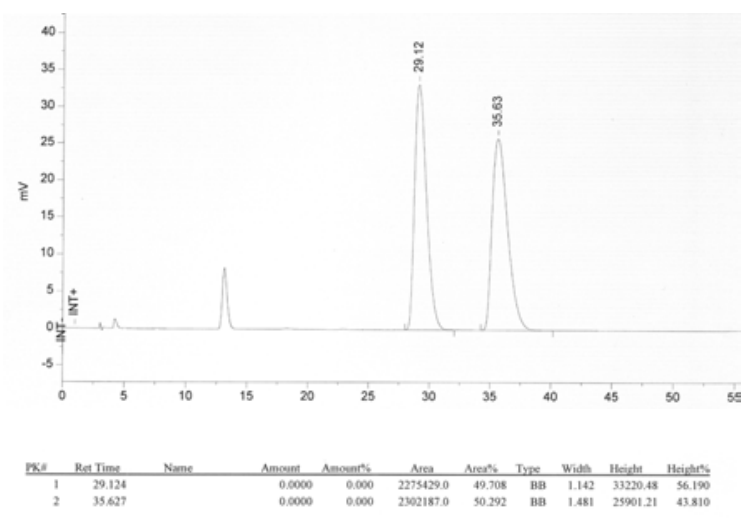

from catalytic reaction

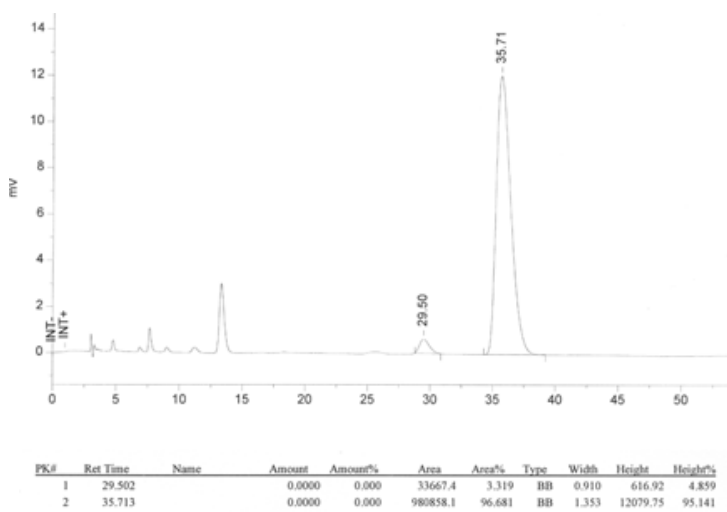

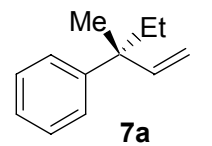

$(\boldsymbol{R})-(-)-3-M e t h y l-3-p h e n y l-1-p e n t e n e . \quad I R \quad(n e a t): 3081(\mathrm{w}), 3056(\mathrm{w})$, $3025(\mathrm{w}), 2968(\mathrm{~s}), 2924(\mathrm{~m}), 2880(\mathrm{~m}), 1646(\mathrm{w}), 1596(\mathrm{w}), 1495(\mathrm{~m})$, $1445(\mathrm{~m}), 1382(\mathrm{~m}), 998(\mathrm{~m}), 910(\mathrm{~s}), 765(\mathrm{~s}), 708(\mathrm{~s}) \mathrm{cm}^{-1} .{ }^{1} \mathrm{H}$ NMR $(400$ $\left.\mathrm{MHz}, \mathrm{CDCl}_{3}\right): \delta$ 7.34-7.17 (5H, m, $\left.\mathrm{ArH}\right), 6.03\left(1 \mathrm{H}, \mathrm{dd}, J=17.6,10.8 \mathrm{~Hz}, \mathrm{CCH}=\mathrm{CH}_{2}\right)$, $5.11\left(1 \mathrm{H}, \mathrm{dd}, J=10.8,1.6 \mathrm{~Hz}, \mathrm{CCH}=\mathrm{CH}_{2}\right), 5.05\left(1 \mathrm{H}, \mathrm{dd}, J=17.6,1.6 \mathrm{~Hz}, \mathrm{CCH}=\mathrm{CH}_{2}\right)$, 1.89-1.72 $\left(2 \mathrm{H}, \mathrm{m}, \mathrm{CCH}_{2} \mathrm{CH}_{3}\right), 1.39\left(3 \mathrm{H}, \mathrm{s}, \mathrm{CCH}_{3}\right), 0.77(3 \mathrm{H}, \mathrm{dd}, J=7.6,7.6 \mathrm{~Hz}$, $\left.\mathrm{CCH}_{2} \mathrm{CH}_{3}\right) .{ }^{13} \mathrm{C}$ NMR $\left(100 \mathrm{MHz}, \mathrm{CDCl}_{3}\right): \delta 147.6,147.1,128.2,126.9,125.8,111.9$, 44.7, 33.6, 24.5, 9.1. HRMS Calcd for $\mathrm{C}_{12} \mathrm{H}_{16}$ : 160.1252. Found: 160.1256. $[\alpha]_{\mathrm{D}}{ }^{20}$ $-12.5^{\circ}\left(c=0.800, \mathrm{CHCl}_{3}\right)$ for a $92 \%$ ee sample. Proof of Stereochemistry: literature value $\left([\alpha]_{\mathrm{D}}^{26}+4.55^{\circ}\left(c=1.1, \mathrm{CHCl}_{3}\right)\right)$ is assigned to the $(S)$ enantiomer. ${ }^{5}$

The optical purity is determined by chiral GLC analysis ( $\beta$-dex, $80{ }^{\circ} \mathrm{C}, 15 \mathrm{psi}$ ); chromatograms are illustrated below:

\footnotetext{
${ }^{5}$ A. Yanagisawa, N. Nomura, Y. Yamada, H. Hibino, H. Yamamoto, Synlett. 1995, 841-842.
} 
Authentic racemic

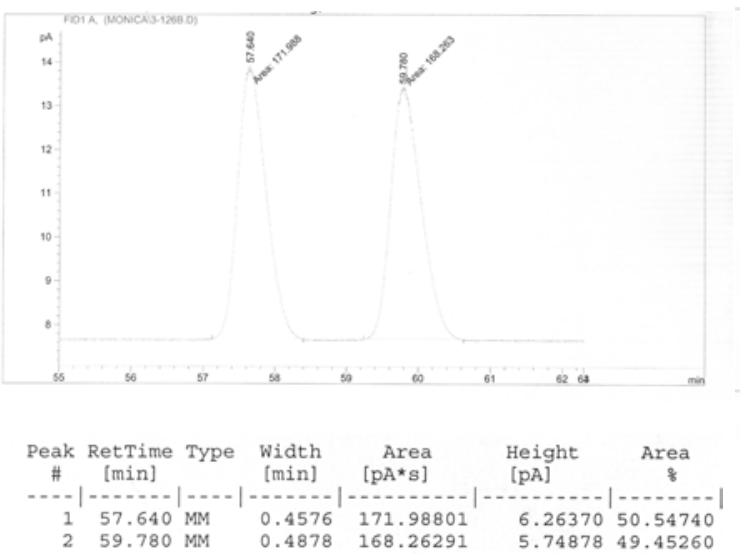

from catalytic reaction

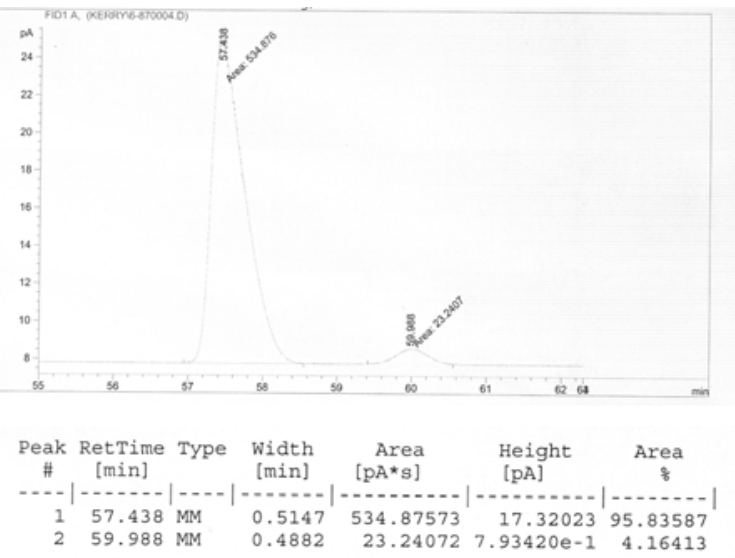

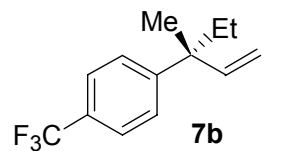

(R)-(-)-3-Methyl-3-(4-trifluoromethylphenyl)-1-pentene. IR (neat): $2974(\mathrm{~m}), 2936$ (m), 2880 (m), 1621 (m), 1337 (s), 1174 (s), 1130 (s), 1073 (s), 1017 (m), 922 (m), 841 (s) $\mathrm{cm}^{-1} .{ }^{1} \mathrm{H}$ NMR (400 MHz, $\left.\mathrm{CDCl}_{3}\right): \delta 7.54(2 \mathrm{H}, \mathrm{d}, J=8.4 \mathrm{~Hz}, \operatorname{ArH}), 7.42(2 \mathrm{H}, \mathrm{dd}, J=8.4,0.8 \mathrm{~Hz}$, $\operatorname{ArH}), 6.00\left(1 \mathrm{H}, \mathrm{dd}, J=17.2,10.8 \mathrm{~Hz}, \mathrm{CCH}=\mathrm{CH}_{2}\right), 5.15(1 \mathrm{H}, \mathrm{dd}, J=10.8,1.2 \mathrm{~Hz}$, $\left.\mathrm{CCH}=\mathrm{CH}_{2}\right), 5.06\left(1 \mathrm{H}, \mathrm{dd}, J=17.2,1.2 \mathrm{~Hz}, \mathrm{CCH}=\mathrm{CH}_{2}\right), 1.88-1.75\left(2 \mathrm{H}, \mathrm{m}, \mathrm{CCH}_{2} \mathrm{CH}_{3}\right)$, $1.37\left(3 \mathrm{H}, \mathrm{s}, \mathrm{CCH}_{3}\right), 0.76\left(3 \mathrm{H}, \mathrm{dd}, J=7.2,7.2 \mathrm{~Hz}, \mathrm{CCH}_{2} \mathrm{CH}_{3}\right) .{ }^{13} \mathrm{C}$ NMR $(100 \mathrm{MHz}$, $\left.\mathrm{CDCl}_{3}\right): \delta 151.8,146.1,127.3,125.1\left(\mathrm{q}, J=3.8 \mathrm{~Hz}, \mathrm{CF}_{3}\right), 123.2,112.8,44.9,33.5,24.5$, 9.0. HRMS Calcd for $\mathrm{C}_{13} \mathrm{H}_{15} \mathrm{~F}_{3}$ : 228.1126. Found: $228.1128 .[\alpha]_{\mathrm{D}}{ }^{20}-14.2^{\circ}(c=1.07$, $\mathrm{CHCl}_{3}$ ) for a $88 \%$ ee sample.

The optical purity is determined by chiral GLC analysis ( $\beta$-dex, $110{ }^{\circ} \mathrm{C}, 15 \mathrm{psi}$ ); chromatograms are illustrated below:

Authentic racemic
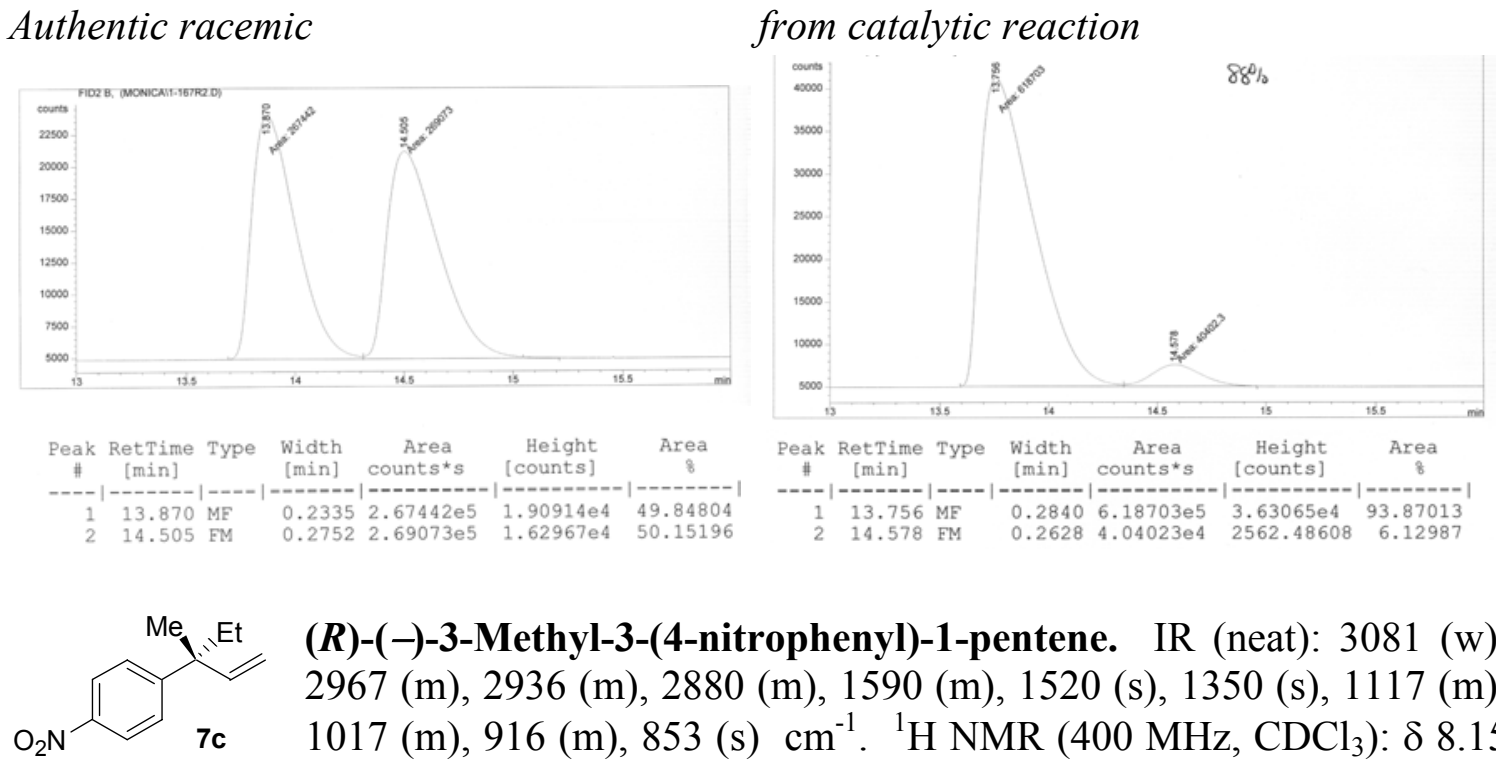

(R)-(-)-3-Methyl-3-(4-nitrophenyl)-1-pentene. IR (neat): 3081 (w), $2967(\mathrm{~m}), 2936(\mathrm{~m}), 2880(\mathrm{~m}), 1590(\mathrm{~m}), 1520(\mathrm{~s}), 1350$ (s), $1117(\mathrm{~m})$, $1017(\mathrm{~m}), 916(\mathrm{~m}), 853(\mathrm{~s}) \mathrm{cm}^{-1} .{ }^{1} \mathrm{H} \mathrm{NMR}\left(400 \mathrm{MHz}, \mathrm{CDCl}_{3}\right): \delta 8.15$ $(2 \mathrm{H}, \mathrm{d}, J=8.8 \mathrm{~Hz}, \mathrm{ArH}), 7.47(2 \mathrm{H}, \mathrm{d}, J=8.8 \mathrm{~Hz}, \mathrm{ArH}), 6.00(1 \mathrm{H}, \mathrm{dd}, J$ $\left.=17.6,10.8 \mathrm{~Hz}, \mathrm{CCH}=\mathrm{CH}_{2}\right), 5.18\left(1 \mathrm{H}, \mathrm{dd}, J=10.8,1.2 \mathrm{~Hz}, \mathrm{CCH}=\mathrm{CH}_{2}\right), 5.08(1 \mathrm{H}, \mathrm{dd}, J$ 
$\left.=17.6,1.2 \mathrm{~Hz}, \mathrm{CCH}=\mathrm{CH}_{2}\right), 1.91-1.75\left(2 \mathrm{H}, \mathrm{m}, \mathrm{CCH}_{2} \mathrm{CH}_{3}\right), 1.39\left(3 \mathrm{H}, \mathrm{s}, \mathrm{CCH}_{3}\right), 0.77(3 \mathrm{H}$, dd, $\left.J=7.6,7.6 \mathrm{~Hz}, \mathrm{CCH}_{2} \mathrm{CH}_{3}\right) .{ }^{13} \mathrm{C} \mathrm{NMR}\left(100 \mathrm{MHz}, \mathrm{CDCl}_{3}\right): \delta 155.5,145.4,127.8$, 123.4, 112.4, 45.3, 33.6, 24.5, 8.9. Anal Calcd for $\mathrm{C}_{12} \mathrm{H}_{15} \mathrm{NO}_{2}$ : C, 70.22; H, 7.37; N, 6.82. Found: $\mathrm{C}, 70.21 ; \mathrm{H}, 7.16 ; \mathrm{N}, 6.67 .[\alpha]_{\mathrm{D}}{ }^{20}-25.1^{\circ}\left(c=1.13, \mathrm{CHCl}_{3}\right)$ for a $87 \%$ ee sample.

The optical purity is determined by chiral GLC analysis ( $\beta$-dex, $130{ }^{\circ} \mathrm{C}, 15 \mathrm{psi}$ ); chromatograms are illustrated below:

Authentic racemic

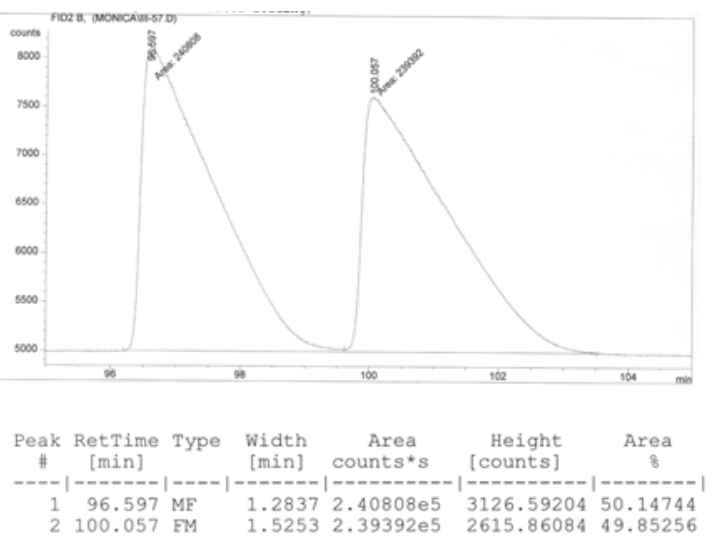

from catalytic reaction

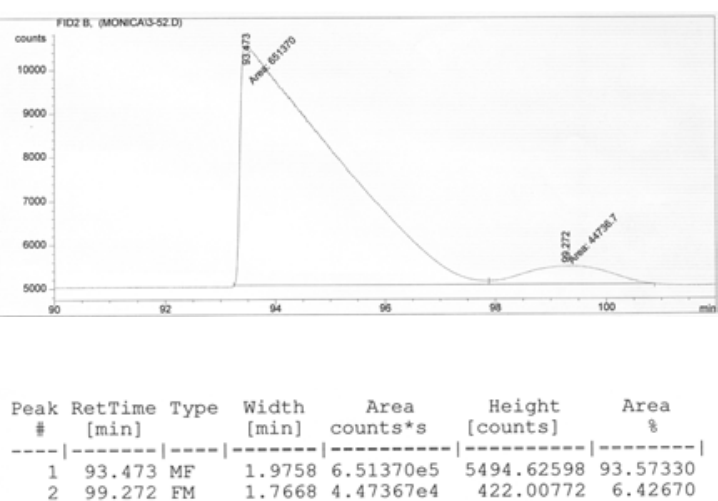

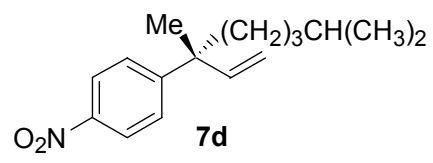
$(2 \mathrm{H}, \mathrm{d}, J=9.2, \mathrm{~Hz}, \mathrm{ArH}), 6.00\left(1 \mathrm{H}, \mathrm{dd}, J=17.6,10.8 \mathrm{~Hz}, \mathbf{C C H}=\mathrm{CH}_{2}\right), 5.17(1 \mathrm{H}, \mathrm{dd}, J=$ $\left.10.8,1.2 \mathrm{~Hz}, \mathrm{CCH}=\mathrm{CH}_{2}\right), 5.07\left(1 \mathrm{H}, \mathrm{dd}, J=17.6,1.2 \mathrm{~Hz}, \mathrm{CCH}=\mathrm{CH}_{2}\right), 1.80-1.68(2 \mathrm{H}, \mathrm{m}$, aliphatic $\mathrm{CH}), 1.50-1.46(1 \mathrm{H}, \mathrm{m}$, aliphatic $\mathrm{CH}), 1.40\left(3 \mathrm{H}, \mathrm{s}, \mathrm{CCH}_{3}\right), 1.19-1.06(4 \mathrm{H}, \mathrm{br} \mathrm{m}$, aliphatic $\mathrm{CH}), 0.81\left(6 \mathrm{H}, \mathrm{d}, J=6.4 \mathrm{~Hz}, \mathrm{CH}\left(\mathrm{CH}_{3}\right)_{2}\right) .{ }^{13} \mathrm{C} \mathrm{NMR}\left(100 \mathrm{MHz}, \mathrm{CDCl}_{3}\right): \delta$ $155.8,145.7,127.7,123.4,113.2,45.1,41.4,39.6,27.9,25.1,22.7,22.6,22.3$. Anal Calcd for $\mathrm{C}_{16} \mathrm{H}_{23} \mathrm{NO}_{2}$ : C, 73.53; H, 8.87; N, 5.36. Found: C, 73.76; H, 8.68; N, 5.26. $[\alpha]_{\mathrm{D}}^{20}-13.0^{\circ}\left(c=1.13, \mathrm{CHCl}_{3}\right)$ for a $83 \%$ ee sample.

The optical purity is determined by chiral HPLC analysis (Chiral Technologies Chiralpak AS, $254 \mathrm{~nm}, 100 \%$ hexanes, $0.5 \mathrm{~mL} / \mathrm{min}, t_{\text {major }}=21 \mathrm{~min}, t_{\text {minor }}=24 \mathrm{~min}$ ).

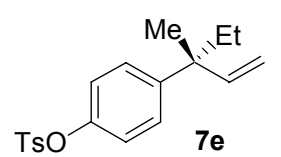

$(\boldsymbol{R})$-(-)-3-Methyl-3-(4-tolylsulfonyloxyphenyl)-1-pentene. IR (neat): $2962(\mathrm{~m}), 2924(\mathrm{~m}), 2873(\mathrm{w}), 1596(\mathrm{~m}), 1501(\mathrm{~s}), 1376(\mathrm{~s}), 1180(\mathrm{~s})$, 1155 (s), 1099 (m), $1023(\mathrm{~m}), 872(\mathrm{~s}) \mathrm{cm}^{-1}$. ${ }^{1} \mathrm{H}$ NMR $(400 \mathrm{MHz}$, $\left.\mathrm{CDCl}_{3}\right): \delta 7.70(2 \mathrm{H}, \mathrm{d}, J=8.4, \mathrm{~Hz}, \operatorname{ArH}), 7.30(2 \mathrm{H}, \mathrm{d}, J=8.4 \mathrm{~Hz}$, $\operatorname{ArH}), 7.21(2 \mathrm{H}, \mathrm{d}, J=8.8, \mathrm{~Hz}, \operatorname{ArH}), 6.89(2 \mathrm{H}, \mathrm{d}, J=8.8 \mathrm{~Hz}, \operatorname{ArH}), 5.94(1 \mathrm{H}, \mathrm{dd}, J=$ $\left.17.6,10.8 \mathrm{~Hz}, \mathrm{CCH}=\mathrm{CH}_{2}\right), 5.09\left(1 \mathrm{H}, \mathrm{dd}, J=10.8,1.6 \mathrm{~Hz}, \mathrm{CCH}=\mathrm{CH}_{2}\right), 4.99(1 \mathrm{H}, \mathrm{dd}, J=$ 17.6, $\left.1.2 \mathrm{~Hz}, \mathrm{CCH}=\mathrm{CH}_{2}\right), 2.44\left(3 \mathrm{H}, \mathrm{s}, \mathrm{ArCH}_{3}\right), 1.82-1.65\left(2 \mathrm{H}, \mathrm{m}, \mathrm{CCH}_{2} \mathrm{CH}_{3}\right), 1.30(3 \mathrm{H}$, s, $\left.\mathrm{CCH}_{3}\right), 0.72\left(3 \mathrm{H}, \mathrm{dd}, J=7.6,7.6 \mathrm{~Hz}, \mathrm{CCH}_{2} \mathrm{CH}_{3}\right) .{ }^{13} \mathrm{C} \mathrm{NMR}\left(100 \mathrm{MHz}, \mathrm{CDCl}_{3}\right): \delta$ 
147.7, 146.6, 146.4, 145.3, 132.8, 129.9, 128.6, 128.1, 121.8, 112.3, 44.5, 33.6, 24.5, 21.8, 8.9. HRMS Calcd for $\mathrm{C}_{19} \mathrm{H}_{22} \mathrm{O}_{3} \mathrm{~S}: 330.1290$. Found: 330.1294 . $[\alpha]_{\mathrm{D}}{ }^{20}-15.4^{\mathrm{o}}(c=$ $1.00, \mathrm{CHCl}_{3}$ ) for a $88 \%$ ee sample.

The optical purity is determined by chiral GLC analysis ( $\beta$-dex, $120{ }^{\circ} \mathrm{C}, 15 \mathrm{psi}$ ) of the derived acetate, obtained by deprotection of the tosyl moiety, and subsequent acylation; chromatograms are illustrated below:

Authentic racemic

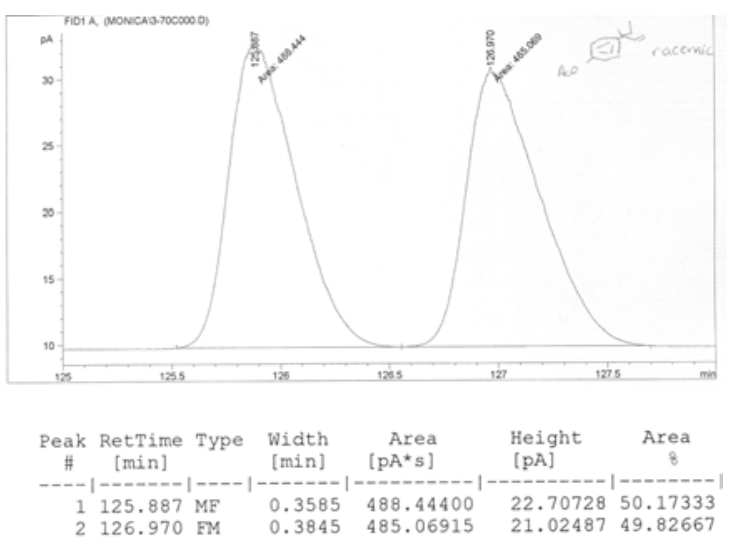

from catalytic reaction

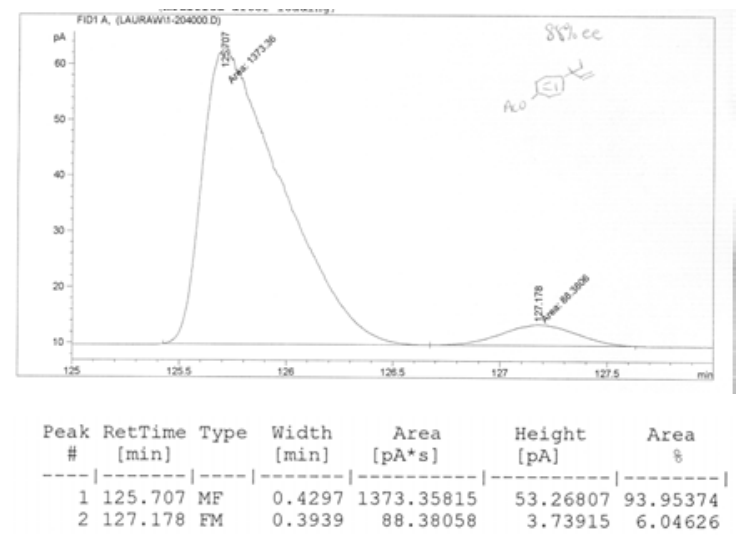

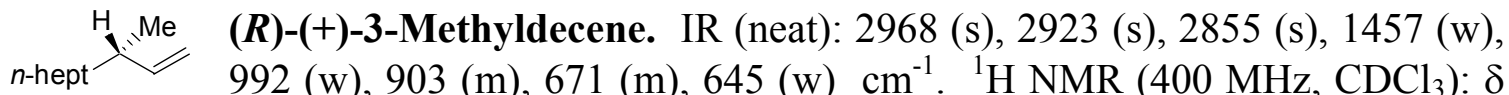
9a $\quad 5.70\left(1 \mathrm{H}, \mathrm{ddd}, J=17.6,10.4,7.6 \mathrm{~Hz}, \mathrm{CHCH}=\mathrm{CH}_{2}\right), 4.97-4.88(2 \mathrm{H}, \mathrm{m}$, $\left.\mathrm{CHCH}=\mathrm{CH}_{2}\right), 2.12-2.08\left(1 \mathrm{H}, \mathrm{m}, \mathrm{CHCH}=\mathrm{CH}_{2}\right), 1.30-1.26(12 \mathrm{H}, \mathrm{br} \mathrm{m}$, aliphatic $\mathrm{CH})$, $0.98\left(3 \mathrm{H}, \mathrm{d}, J=6.8 \mathrm{~Hz}, \mathrm{CHCH}_{3}\right), 0.88\left(3 \mathrm{H}, \mathrm{dd}, J=6.8,6.8 \mathrm{~Hz}, \mathrm{CH}_{2} \mathrm{CH}_{2} \mathrm{CH}_{3}\right) .{ }^{13} \mathrm{C} \mathrm{NMR}$ $\left(100 \mathrm{MHz}, \mathrm{CDCl}_{3}\right): \delta 145.2,112.3,37.9,36.9,32.1,29.9,29.5,27.4,22.8,20.3,14.3$. HRMS Calcd for $\mathrm{C}_{11} \mathrm{H}_{22}$ : 154.1722. Found: 154.1715. $[\alpha]_{\mathrm{D}}^{20}+9.4^{\circ}\left(c=0.53, \mathrm{CHCl}_{3}\right)$ for a $78 \%$ ee sample.

The optical purity is determined by chiral GLC analysis (Chiraldex-GTA,
$32 \mathrm{O}$
$60{ }^{\circ} \mathrm{C}, 15 \mathrm{psi}$ of the derived ketone 26, obtained by Wacker ${ }^{6}$ oxidation: $\left.\mathrm{CHCHCH}_{3}\right), 2.13\left(3 \mathrm{H}, \mathrm{s}, \mathrm{COCH}_{3}\right), 1.68-1.60(1 \mathrm{H}, \mathrm{m}$, aliphatic $\mathrm{CH}), 1.38-1.21(11 \mathrm{H}, \mathrm{br}$ m, aliphatic CH), $1.07\left(3 \mathrm{H}, \mathrm{d}, J=7.2 \mathrm{~Hz}, \mathrm{CHCH}_{3}\right), 0.88(3 \mathrm{H}, \mathrm{dd}, J=7.2,7.2 \mathrm{~Hz}$, $\mathrm{CH}_{2} \mathrm{CH}_{2} \mathrm{CH}_{3}$ ); chromatograms are illustrated below:

\footnotetext{
${ }^{6}$ a) Y. Ito, T. Hirao, T. Saegusa, J. Org. Chem. 1978, 43, 1011-1013. b) S. J. Degrado, Mizutani, H, A. H. Hoveyda, J. Am. Chem. Soc. 2001, 123, 755-756.
} 
Authentic racemic

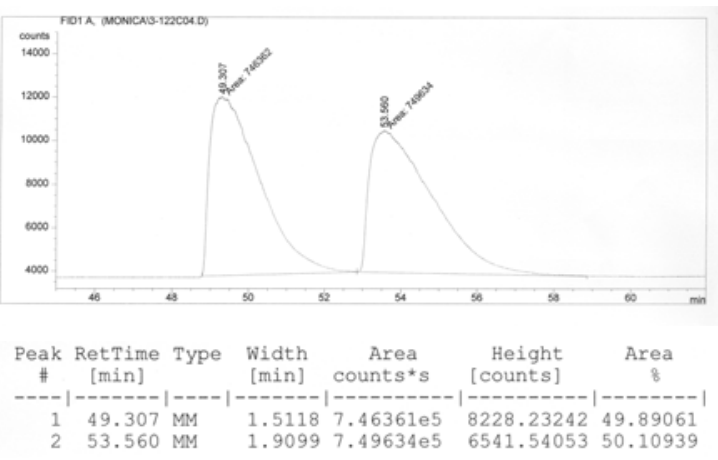

from catalytic reaction

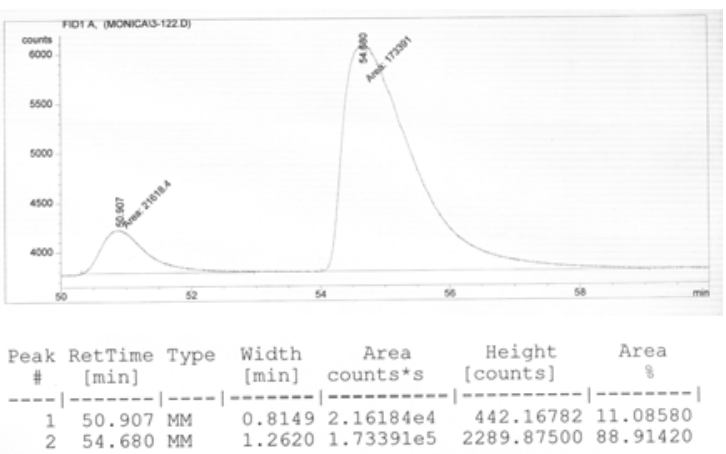

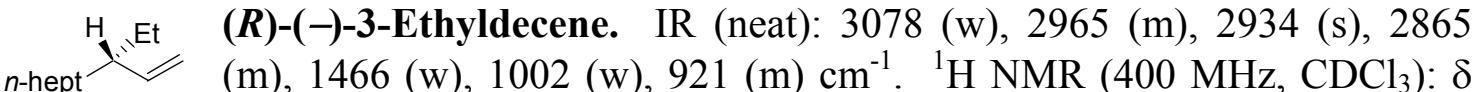
9b $\quad 5.52\left(1 \mathrm{H}\right.$, ddd, $\left.J=16.8,10.4,8.4 \mathrm{~Hz}, \mathrm{CHCH}=\mathrm{CH}_{2}\right), 4.98-4.91(2 \mathrm{H}, \mathrm{m}$, $\left.\mathrm{CHCH}=\mathrm{CH}_{2}\right), 1.85-1.83\left(1 \mathrm{H}, \mathrm{m}, \mathrm{CHCH}=\mathrm{CH}_{2}\right), 1.43-1.19(14 \mathrm{H}, \mathrm{br} \mathrm{m}$, aliphatic $\mathrm{CH}), 0.88\left(3 \mathrm{H}, \mathrm{dd}, J=6.8,6.8 \mathrm{~Hz}, \mathrm{CH}_{2} \mathrm{CH}_{3}\right), 0.84(3 \mathrm{H}, \mathrm{dd}, J=7.6,7.6 \mathrm{~Hz}$, $\left.\mathrm{CH}_{2} \mathrm{CH}_{3}\right) .{ }^{13} \mathrm{C}$ NMR $\left(100 \mathrm{MHz}, \mathrm{CDCl}_{3}\right): \delta$ 143.6, 114.1, 46.0, 34.8, 32.1, 29.9, 29.5, 27.8, 27.4, 22.8, 14.3, 11.8. Anal Calcd for $\mathrm{C}_{12} \mathrm{H}_{24}: \mathrm{C}, 85.63 ; \mathrm{H}, 14.37$. Found: $\mathrm{C}$, 85.29; $\mathrm{H}, 14.61$. $[\alpha]_{\mathrm{D}}{ }^{20}-2.0^{\circ}\left(c=0.80, \mathrm{CHCl}_{3}\right)$ for a $92 \%$ ee sample.

The optical purity is determined by chiral GLC analysis ( $\beta$-dex, $\left.100{ }^{\circ} \mathrm{C}, 15 \mathrm{psi}\right)$ of the derived alcohol, obtained by ozonolysis followed by $\mathrm{NaBH}_{4}$ reduction; chromatograms are illustrated below:

Authentic racemic
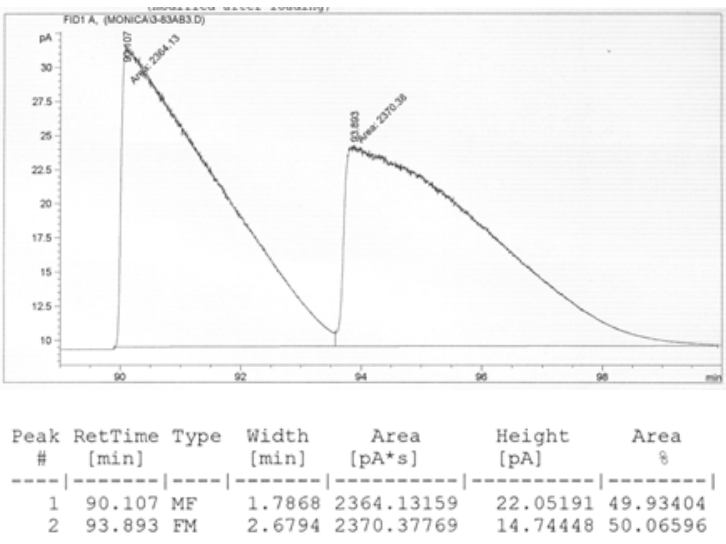

\section{from catalytic reaction}

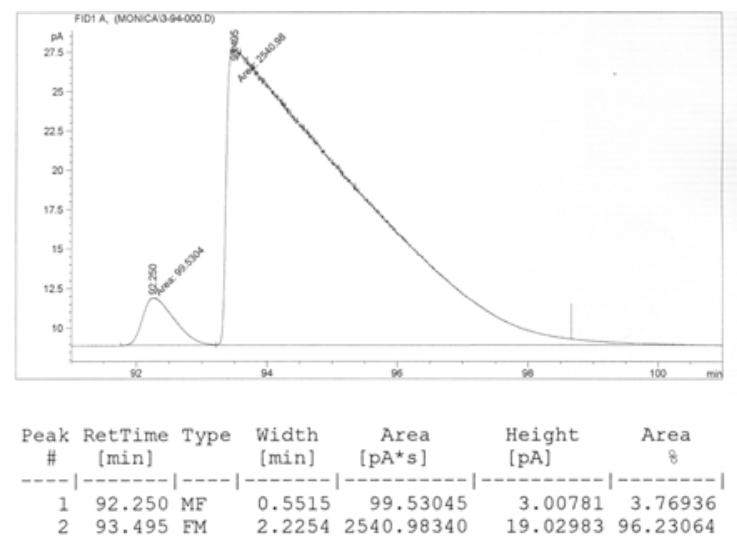

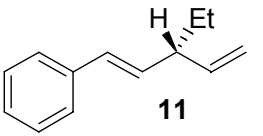

(R)-(-)-3-ethyl-1-phenyl-1,4-pentadiene (5.7:1 mixture of $S_{\mathrm{N}} 2$ ' to $\mathbf{S}_{\mathrm{N}} 2$ products). IR (neat): $3081(\mathrm{~m}), 3069(\mathrm{~m}), 3025(\mathrm{~m}), 2967(\mathrm{~s})$, $2924(\mathrm{~m}), 2886(\mathrm{~m}), 1646(\mathrm{~m}), 1596(\mathrm{~s}), 1495(\mathrm{~m}), 1457(\mathrm{~m}), 992(\mathrm{~s})$, 966 (s), 929 (s), 765 (s), 690 (m) cm ${ }^{-1} .{ }^{1} \mathrm{H}$ NMR $\left(400 \mathrm{MHz}, \mathrm{CDCl}_{3}\right): \delta 7.34-7.28(4 \mathrm{H}, \mathrm{m}$, $\operatorname{ArH}), 7.22-7.18(1 \mathrm{H}, \mathrm{m}, \operatorname{ArH}), 6.39(1 \mathrm{H}, \mathrm{d}, J=16.0 \mathrm{~Hz}, \operatorname{ArCH}), 6.12(1 \mathrm{H}, \mathrm{dd}, J=16.0$, 
7.6 Hz, ArCHCH), $5.80\left(1 \mathrm{H}, \mathrm{ddd}, J=17.6,10.4,7.2 \mathrm{~Hz}, \mathrm{CHCH}=\mathrm{CH}_{2}\right), 5.10-5.03(2 \mathrm{H}$, $\left.\mathrm{m}, \mathrm{CHCH}=\mathrm{CH}_{2}\right), 2.77\left(1 \mathrm{H}, \mathrm{ddd}, J=7.2,7.2,7.2 \mathrm{~Hz}, \mathrm{CHCH}=\mathrm{CH}_{2}\right), 1.57-1.43(2 \mathrm{H}, \mathrm{m}$, $\left.\mathrm{CHCH}_{2} \mathrm{CH}_{3}\right), 0.93\left(3 \mathrm{H}, \mathrm{dd}, J=7.2,7.2 \mathrm{~Hz}, \mathrm{CHCH}_{2} \mathrm{CH}_{3}\right) .{ }^{13} \mathrm{C} \mathrm{NMR}\left(100 \mathrm{MHz}, \mathrm{CDCl}_{3}\right)$ : $\delta$ 141.4, 137.9, 133.2, 129.8, 128.6, 127.1, 126.2, 114.5, 49.0, 27.9, 11.9. HRMS Calc for $\mathrm{C}_{12} \mathrm{H}_{20}$ : 164.1567. Found: 164.1566 . $[\alpha]_{\mathrm{D}}{ }^{20}-50.1^{\circ}\left(c=0.810, \mathrm{CHCl}_{3}\right)$ for a $89 \%$ ee sample.

The optical purity is determined by chiral GLC analysis ( $\beta$-dex, $80{ }^{\circ} \mathrm{C}, 20 \mathrm{psi}$ ); chromatograms are illustrated below:

Authentic racemic

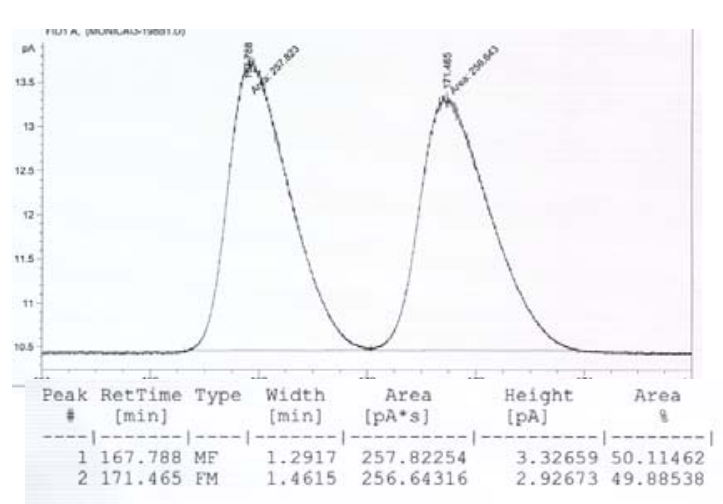

from catalytic reaction

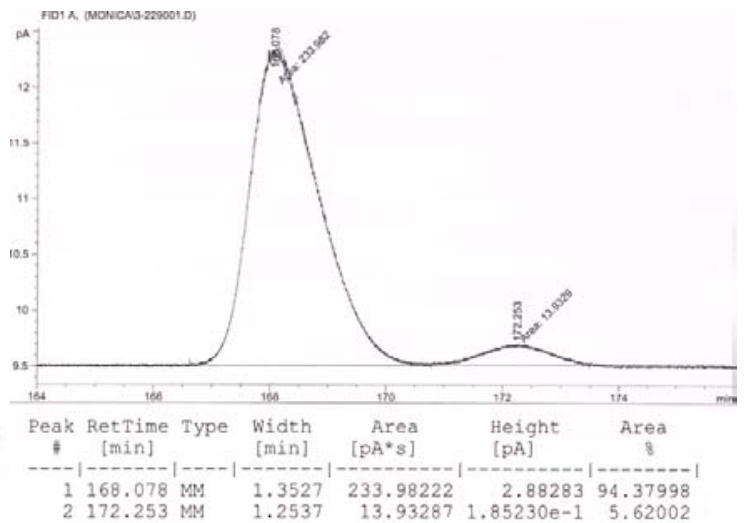

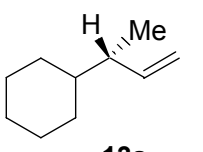

$13 a$

(R)-(+)-3-Cyclohexyl-1-butene. IR (neat): 2924 (s), 2855 (m), 910 (m), $790(\mathrm{w}), 677(\mathrm{~m}), 652(\mathrm{~s}) \mathrm{cm}^{-1} .{ }^{1} \mathrm{H}$ NMR $\left(400 \mathrm{MHz}, \mathrm{CDCl}_{3}\right): \delta 5.52(1 \mathrm{H}$, ddd, $\left.J=17.6,9.6,8.0 \mathrm{~Hz}, \mathrm{CHCH}=\mathrm{CH}_{2}\right), 4.95-4.89\left(2 \mathrm{H}, \mathrm{m}, \mathrm{CHCH}=\mathrm{CH}_{2}\right)$, $1.95\left(1 \mathrm{H}\right.$, ddq, $\left.J=6.8,6.8,6.8 \mathrm{~Hz}, \mathrm{CHCH}=\mathrm{CH}_{2}\right), 1.74-1.60(5 \mathrm{H}, \mathrm{br} \mathrm{m}$, aliphatic $\mathrm{CH}), 1.26-1.01(4 \mathrm{H}$, br m, aliphatic $\mathrm{CH}), 0.96\left(3 \mathrm{H}, \mathrm{d}, J=6.8 \mathrm{~Hz}, \mathrm{CHCH}_{3}\right)$, 0.99-0.87 (2H, m, aliphatic CH). ${ }^{13} \mathrm{C}$ NMR $\left(100 \mathrm{MHz}, \mathrm{CDCl}_{3}\right): \delta 143.8,113.1,43.7$, 43.0, 30.5, 30.4, 26.8, 26.8, 17.2. Anal Calcd for $\mathrm{C}_{10} \mathrm{H}_{18}: \mathrm{C}, 86.88 ; \mathrm{H}, 13.12$. Found: $\mathrm{C}$, 86.65; H, 13.33. HRMS Calcd for $\mathrm{C}_{10} \mathrm{H}_{18}: 138.1409$. Found: $138.1406 .[\alpha]_{\mathrm{D}}{ }^{20}+13.6^{\circ}(c$ $=0.500, \mathrm{CHCl}_{3}$ ) for a $87 \%$ ee sample.

The optical purity of the compound is determined by chiral GLC analysis $\left(\beta-\operatorname{dex}, 100{ }^{\circ} \mathrm{C}\right.$, 15 psi); chromatograms are illustrated below:

Authentic racemic

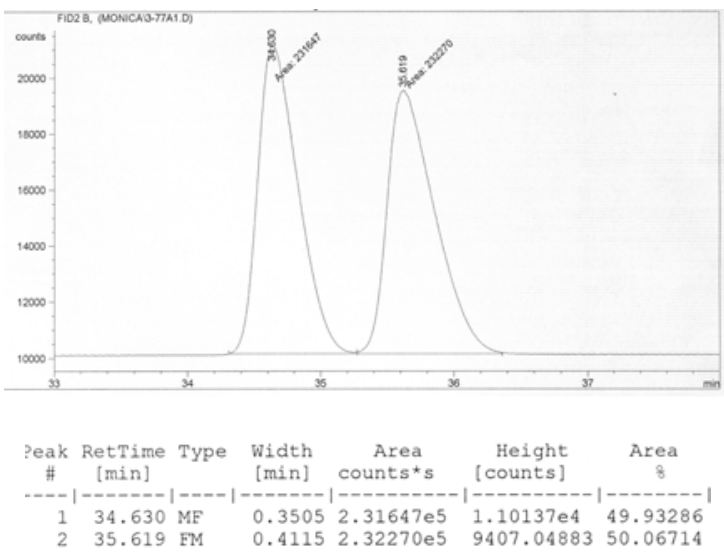

from catalytic reaction

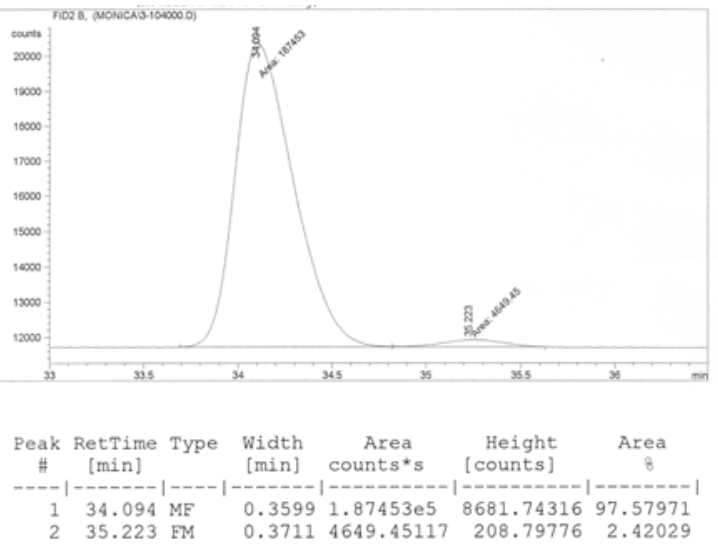




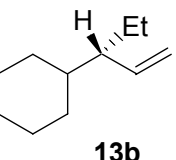

(R)-(-)-3-Cyclohexyl-1-pentene. IR (neat): 2930 (m), 2855 (m), 992 (w), $910(\mathrm{~m}), 678(\mathrm{~s}), 652(\mathrm{~s}) \mathrm{cm}^{-1} .{ }^{1} \mathrm{H}$ NMR $\left(400 \mathrm{MHz}, \mathrm{CDCl}_{3}\right): \delta 5.52(1 \mathrm{H}$, ddd, $\left.J=16.8,9.6,9.2 \mathrm{~Hz}, \mathrm{CHCH}=\mathrm{CH}_{2}\right), 4.99-4.86\left(2 \mathrm{H}, \mathrm{m}, \mathrm{CHCH}=\mathrm{CH}_{2}\right)$, 1.71-1.61 (6H, br m, aliphatic CH), $1.46(1 \mathrm{H}, \mathrm{dqd}, J=13.3,7.4,4.1 \mathrm{~Hz}$, $\left.\mathrm{CHCHHCH}_{3}\right), 1.26-0.84(7 \mathrm{H}$, br $\mathrm{m}$, aliphatic $\mathrm{CH}), 0.81(3 \mathrm{H}, \mathrm{dd}, J=7.6,7.6 \mathrm{~Hz}$, $\left.\mathrm{CHCH}_{2} \mathrm{CH}_{3}\right) .{ }^{13} \mathrm{C} \mathrm{NMR}\left(100 \mathrm{MHz}, \mathrm{CDCl}_{3}\right): \delta 141.6,115.1,52.2,41.7,31.3,29.9,26.9$, 26.9, 26.9, 24.5, 12.2. Anal Calcd for $\mathrm{C}_{11} \mathrm{H}_{20}$ : C, 86.76; H, 13.24. Found: $\mathrm{C}, 86.40 ; \mathrm{H}$, 13.50. $[\alpha]_{20}{ }^{\mathrm{D}}-9.2^{\circ}\left(c=0.80, \mathrm{CHCl}_{3}\right)$ for a $95 \%$ ee sample.

The optical purity of the compound is determined by chiral GLC analysis $\left(\alpha-\operatorname{dex}, 60{ }^{\circ} \mathrm{C}\right.$, 15 psi); chromatograms are illustrated below:

Authentic racemic

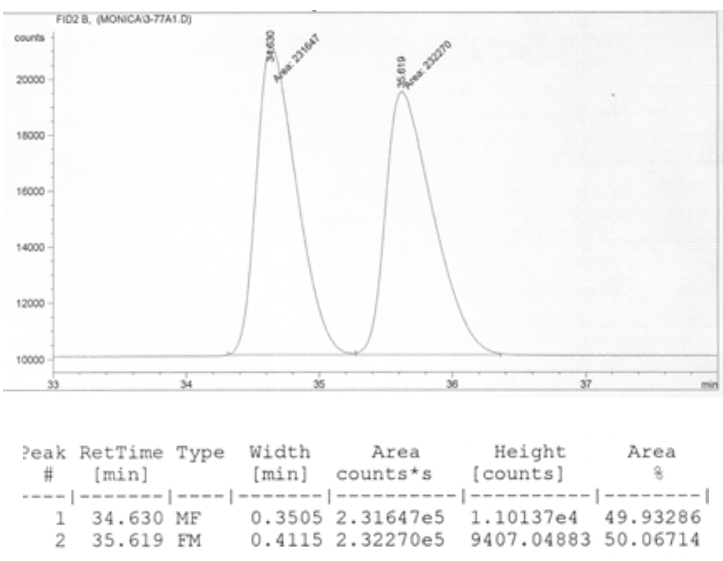

from catalytic reaction

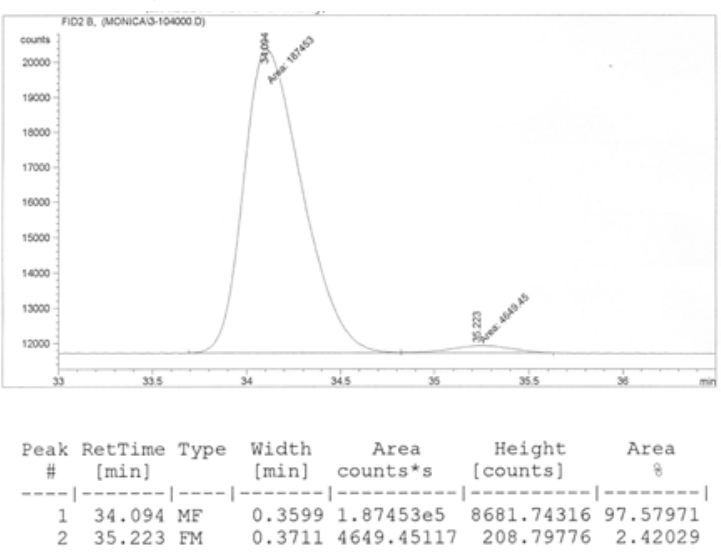

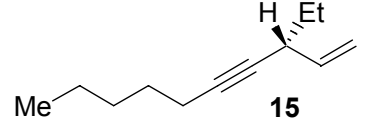
${ }^{1} \mathrm{H}$ NMR (400 MHz, $\left.\mathrm{CDCl}_{3}\right): \delta 5.76\left(1 \mathrm{H}, \mathrm{ddd}, J=16.8,10.0,6.0 \mathrm{~Hz}, \mathrm{CHCH}=\mathrm{CH}_{2}\right), 5.28$ $(1 \mathrm{H}, \mathrm{ddd}, J=16.8,1.8,1.8 \mathrm{~Hz}, \mathrm{CHCH}=\mathrm{CHH}), 5.05(1 \mathrm{H}, \mathrm{ddd}, J=16.8,1.8,1.8 \mathrm{~Hz}$, $\mathrm{CHCH}=\mathrm{CHH}), 3.00-2.94\left(1 \mathrm{H}, \mathrm{m}, \mathrm{CHCH}=\mathrm{CH}_{2}\right), 2.20(2 \mathrm{H}, \mathrm{ddd}, J=9.2,6.8,2.0$, $\left.\mathrm{CH}_{2} \mathrm{CC}\right), 1.62-1.26(8 \mathrm{H}, \mathrm{m}$, aliphatic $\mathrm{CH}), 0.98\left(3 \mathrm{H}, \mathrm{dd}, J=7.2,7.2 \mathrm{~Hz}, \mathrm{CH}_{2} \mathrm{CH}_{3}\right), 0.90$ $\left(3 \mathrm{H}, \mathrm{dd}, J=7.2,7.2 \mathrm{~Hz}, \mathrm{CH}_{2} \mathrm{CH}_{3}\right) .{ }^{13} \mathrm{C} \mathrm{NMR}\left(100 \mathrm{MHz}, \mathrm{CDCl}_{3}\right): \delta 139.0,114.7,83.8$, 80.5, 37.3, 31.2, 29.0, 28.8, 22.4, 18.9, 14.2, 11.6. HRMS Calc for $\mathrm{C}_{13} \mathrm{H}_{16}$ : 172.1248 . Found: $172.1252 .[\alpha]_{\mathrm{D}}{ }^{20}-26.2^{\circ}\left(c=0.600, \mathrm{CHCl}_{3}\right)$ for a $96 \%$ ee sample.

The optical purity is determined by chiral GLC analysis ( $\beta$-dex, $58{ }^{\circ} \mathrm{C}, 15 \mathrm{psi}$ ); chromatograms are illustrated below: 
Authentic racemic
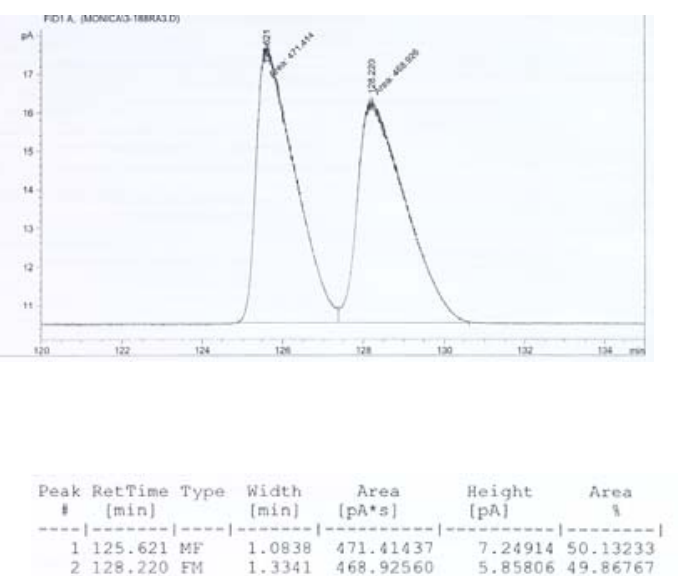

from catalytic reaction
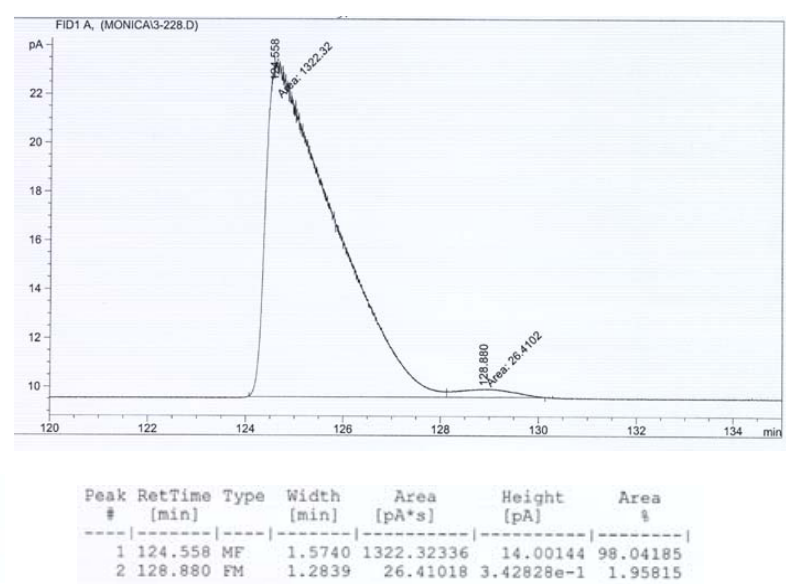

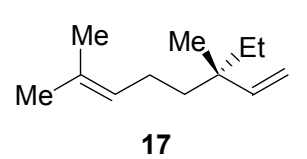

(R)-(-)-3-Ethyl-3,7-dimethyl-1,6-octadiene. IR (neat): 3078 (w), $2965(\mathrm{~s}), 2915(\mathrm{~s}), 2877(\mathrm{~m}), 2642(\mathrm{w}), 1460(\mathrm{~m}), 1385(\mathrm{~m}), 1002(\mathrm{~m})$, $914(\mathrm{~s}) \mathrm{cm}^{-1} .{ }^{1} \mathrm{H}$ NMR $\left(400 \mathrm{MHz}, \mathrm{CDCl}_{3}\right): \delta 5.68(1 \mathrm{H}, \mathrm{dd}, J=17.2$, $\left.10.8 \mathrm{~Hz}, \mathrm{CCH}=\mathrm{CH}_{2}\right), 5.11-5.07\left(1 \mathrm{H}, \mathrm{m},\left(\mathrm{CH}_{3}\right)_{2} \mathrm{C}=\mathrm{CH}\right), 4.93(2 \mathrm{H}, \mathrm{ddd}$, $\left.\left(\mathrm{CH}_{3}\right)_{2} \mathrm{C}=\mathrm{CH}\right), 1.59\left(3 \mathrm{H}, \mathrm{s},\left(\mathrm{CH}_{3}\right)_{2} \mathrm{C}=\mathrm{CH}\right), 1.36-1.26(4 \mathrm{H}, \mathrm{m}$, aliphatic $\mathrm{CH}), 0.94(3 \mathrm{H}, \mathrm{s}$, $\left.\mathrm{CCH}_{3}\right), 0.79\left(3 \mathrm{H}, \mathrm{d}, J=7.4,7.4 \mathrm{~Hz}, \mathrm{CCH}_{2} \mathrm{CH}_{3}\right) .{ }^{13} \mathrm{C} \mathrm{NMR}\left(100 \mathrm{MHz}, \mathrm{CDCl}_{3}\right): \delta 147.3$, 131.1, 125.3 111.7, 40.6, 39.7, 33.2, 25.8, 23.0, 22.1, 17.7, 8.5. Anal Calcd for $\mathrm{C}_{12} \mathrm{H}_{22}$ : $\mathrm{C}, 86.67 ; \mathrm{H}, 13.33$. Found: $\mathrm{C}, 86.28 ; \mathrm{H}, 13.70 .[\alpha]_{\mathrm{D}}{ }^{20}-18.6^{\circ}\left(c=1.07, \mathrm{CHCl}_{3}\right)$ for a $82 \%$ ee sample.

The optical purity is determined by chiral GLC analysis (Chiraldex-GTA, $80{ }^{\circ} \mathrm{C}, 15 \mathrm{psi}$ ) of the derived diacetate, obtained by ozonolysis followed by $\mathrm{NaBH}_{4}$ reduction and subsequent acylation, $t_{\text {major }}=317 \mathrm{~min}, t_{\text {minor }}=324 \mathrm{~min}$ ).

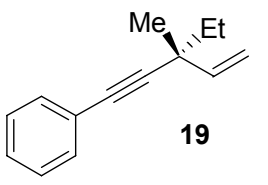

(R)-(-)-1-phenyl-3-ethyl-3-methyl-pent-4-en-1-yne. IR (neat): 3081 (m), 3050 (w), 2974 (s), 2923 (s), $2880(\mathrm{~m}), 1652$ (m), 1615 (m), 1495 (s), $1445(\mathrm{~m}), 1394(\mathrm{~m}), 1344(\mathrm{~m}), 922(\mathrm{~s}), 771(\mathrm{~s}), 690(\mathrm{~s}) \mathrm{cm}^{-1} \cdot{ }^{1} \mathrm{H}$ NMR (400 MHz, $\left.\mathrm{CDCl}_{3}\right): \delta$ 7.45-7.41 (2H, m, ArH), 7.31-7.27 (3H, m, $\operatorname{ArH}), 5.92\left(1 \mathrm{H}, \mathrm{dd}, J=16.8,10.4 \mathrm{~Hz}, \mathrm{CCH}=\mathrm{CH}_{2}\right), 5.45-5.40\left(1 \mathrm{H}, \mathrm{m}, \mathrm{CHCH}=\mathrm{CH}_{2}\right)$, 5.11-5.08 $\left(1 \mathrm{H}, \mathrm{m}, \mathrm{CHCH}=\mathrm{CH}_{2}\right), 1.67-1.53\left(2 \mathrm{H}, \mathrm{m}, \mathrm{CCH}_{2} \mathrm{CH}_{3}\right), 1.36\left(3 \mathrm{H}, \mathrm{s}, \mathrm{CCH}_{3}\right), 1.00$ $\left(3 \mathrm{H}, \mathrm{dd}, J=7.2,7.2 \mathrm{~Hz}, \mathrm{CCH}_{2} \mathrm{CH}_{3}\right) .{ }^{13} \mathrm{C} \mathrm{NMR}\left(100 \mathrm{MHz}, \mathrm{CDCl}_{3}\right): \delta 143.4,131.8$, 128.3, 127.7, 124.1, 113.3, 93.6, 84.0, 40.0, 35.1, 27.8, 9.8. Anal Calcd for $\mathrm{C}_{14} \mathrm{H}_{16}$ : C, 91.25; $\mathrm{H}, 8.75$. Found: $\mathrm{C}, 90.98 ; \mathrm{H}, 9.00 .[\alpha]_{\mathrm{D}}{ }^{20}-31.8^{\circ}\left(c=1.05, \mathrm{CHCl}_{3}\right)$ for a $91 \%$ ee sample.

The optical purity is determined by chiral HPLC analysis (Chiral Technologies Chiralcel OD, $254 \mathrm{~nm}, 98: 2$ hexanes:isopropanol, $1 \mathrm{~mL} / \mathrm{min}$ ) of the derived alcohol, obtained by hydroboration with 9-BBN; chromatograms are illustrated below: 
Authentic racemic

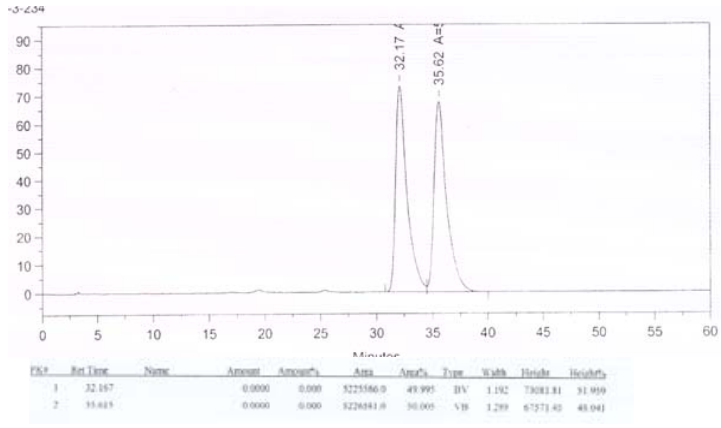

from catalytic reaction

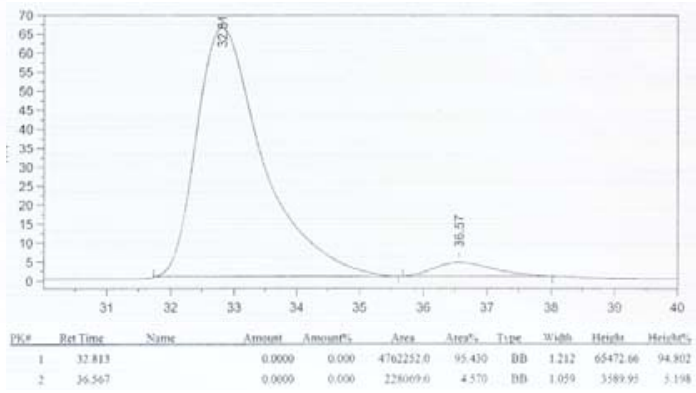

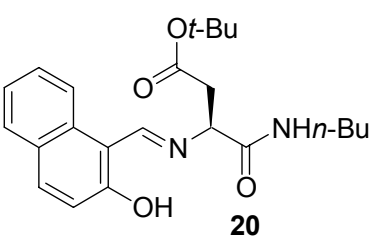

2-Hydroxy-1-napthaldehyde- L-Asp(Ot-Bu)-NHn-Bu: IR (solid film): 3307 (br), 3062 (w), 2968 (m), 2936 (m), 2880 (w), $1741(\mathrm{~m}), 1665(\mathrm{~m}), 1640(\mathrm{~s}), 1539(\mathrm{~m}), 1376(\mathrm{~m}), 1294(\mathrm{~m})$, $1243(\mathrm{~m}), 1155(\mathrm{~s}), 841(\mathrm{~m}), 753(\mathrm{~m}) \mathrm{cm}^{-1}$. ${ }^{1} \mathrm{H}$ NMR $(400 \mathrm{MHz}$, $\left.\mathrm{CDCl}_{3}\right): \delta 9.25(1 \mathrm{H}, \mathrm{s}, \mathbf{H C}=\mathrm{N}), 8.07(1 \mathrm{H}, \mathrm{d}, J=8.0 \mathrm{~Hz}, \mathrm{ArH})$, $7.82(1 \mathrm{H}, \mathrm{d}, J=9.2 \mathrm{~Hz}, \operatorname{ArH}), 7.74(1 \mathrm{H}, \mathrm{d}, J=8.0 \mathrm{~Hz}, \operatorname{ArH})$, $7.53(1 \mathrm{H}, \mathrm{ddd}, J=8.0,8.0,1.2 \mathrm{~Hz}, \operatorname{ArH}), 7.35(1 \mathrm{H}, \mathrm{m}, \operatorname{ArH}), 7.13(1 \mathrm{H}, \mathrm{d}, J=9.2 \mathrm{~Hz}$, ArH), 6.20 (1H, br s, NH), 4.43 (1H, dd, $J=9.2,3.6 \mathrm{~Hz}, \mathrm{NCHCO}), 3.37-3.22(2 \mathrm{H}, \mathrm{m}$, $\left.\mathrm{NCH}_{2}\right), 3.16(1 \mathrm{H}, \mathrm{dd}, J=16.4,3.6 \mathrm{~Hz}, \mathrm{CHCHHCO}), 2.80(1 \mathrm{H}, \mathrm{dd}, J=16.4,9.2 \mathrm{~Hz}$, CHCHHCO), 1.52-1.45 (2H, m, aliphatic CH), $1.36(9 \mathrm{H}, \mathrm{s}, t-\mathbf{B u}), 1.40-1.25(2 \mathrm{H}, \mathrm{m}$, aliphatic $\mathrm{CH}), 0.90\left(3 \mathrm{H}, \mathrm{t}, J=7.2 \mathrm{~Hz}, \mathrm{CH}_{3}\right) .{ }^{13} \mathrm{C} \mathrm{NMR}\left(100 \mathrm{MHz}, \mathrm{CDCl}_{3}\right): \delta 170.2$, $170.0,165.4,165.3,135.9,132.9,129.4,128.3,127.7,123.8,120.5,119.3,108.6,81.6$, 68.9, 40.1, 39.6, 31.7, 28.1, 20.1, 13.8. HRMS Calcd for $\mathrm{C}_{23} \mathrm{H}_{30} \mathrm{~N}_{2} \mathrm{O}_{4} \mathrm{Na}\left(\mathrm{M}+\mathrm{Na}^{+}\right)$: 421.2101. Found: 421.2101.

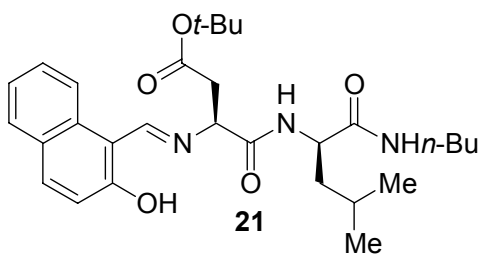

2-Hydroxy-1-napthaldehyde- L-Asp (Ot-Bu)-D-LeuNHn-Bu: IR (solid film): 3283 (br), 3056 (w), 2962 (m), 2936 (m), 1741 (m), 1652 (s), 1552 (m), 1376 (m), 1300 (w), $1256(\mathrm{w}), 1155(\mathrm{~m}), 840(\mathrm{w}), 753(\mathrm{w}) \mathrm{cm}^{-1} \cdot{ }^{1} \mathrm{H}$ NMR $\left(400 \mathrm{MHz}, \mathrm{CDCl}_{3}\right): \delta 9.27(1 \mathrm{H}, \mathrm{s}, \mathbf{H C}=\mathrm{N}), 8.06(1 \mathrm{H}, \mathrm{d}, J$ $=8.4 \mathrm{~Hz}, \operatorname{ArH}), 7.84(1 \mathrm{H}, \mathrm{d}, J=9.2 \mathrm{~Hz}, \operatorname{ArH}), 7.76(1 \mathrm{H}$, d, $J=7.2 \mathrm{~Hz}, \operatorname{ArH}), 7.54(1 \mathrm{H}, \mathrm{ddd}, J=7.2,7.2,1.2 \mathrm{~Hz}, \operatorname{ArH}), 7.37$ (1H, ddd, $J=7.2$, 7.2, $1.2 \mathrm{~Hz}, \operatorname{ArH}), 7.15(1 \mathrm{H}, \mathrm{d}, J=9.2 \mathrm{~Hz}, \operatorname{ArH}), 6.95(1 \mathrm{H}, \mathrm{dd}, J=4.8,4.8 \mathrm{~Hz}, \mathrm{NH})$, $6.45(1 \mathrm{H}, \mathrm{d}, J=8.4 \mathrm{~Hz}, \mathrm{NH}), 4.53-4.47(1 \mathrm{H}, \mathrm{m}, \mathrm{NCHCO}), 4.35(1 \mathrm{H}, \mathrm{dd}, J=4.8,4.8 \mathrm{~Hz}$, $\mathrm{NCHCO}), 3.32-3.18\left(2 \mathrm{H}, \mathrm{m}, \mathrm{NCH}_{2}\right), 3.18$ (1H, dd, $\left.J=16.4,5.6 \mathrm{~Hz}, \mathrm{CHCHHCO}\right), 2.88$ $(1 \mathrm{H}, \mathrm{dd}, J=16.4,4.8 \mathrm{~Hz}, \mathrm{CHCHHCO}), 1.87-1.81(1 \mathrm{H}, \mathrm{m}$, aliphatic CH), 1.58-1.45 (4H, $\mathrm{m}$, aliphatic CH), $1.42(9 \mathrm{H}, \mathrm{s}, t$-Bu), $1.33(2 \mathrm{H}$, ddddd, $J=7.2,7.2,7.2,7.2,7.2 \mathrm{~Hz}$, aliphatic $\mathrm{CH}), 0.91\left(3 \mathrm{H}, \mathrm{dd}, J=7.2,7.2 \mathrm{~Hz}, \mathrm{CH}_{3}\right), 0.87\left(3 \mathrm{H}, \mathrm{d}, J=6.4 \mathrm{~Hz}, \mathrm{CH}\left(\mathrm{CH}_{3}\right)_{2}\right)$, $0.84\left(3 \mathrm{H}, \mathrm{d}, J=6.4 \mathrm{~Hz}, \mathrm{CH}\left(\mathrm{CH}_{3}\right)_{2}\right) .{ }^{13} \mathrm{C} \mathrm{NMR}\left(100 \mathrm{MHz}, \mathrm{CDCl}_{3}\right): \delta 171.3,170.6,170.4$, $164.8,164.5,136.0,132.8,129.4,128.3,127.8,123.9,120.3,119.2,108.6,82.2,68.7$, 52.3, 40.6, 40.0, 39.6, 31.7, 28.1, 25.2, 23.1, 21.9, 20.2, 13.9. HRMS Calcd for $\mathrm{C}_{29} \mathrm{H}_{41} \mathrm{~N}_{3} \mathrm{O}_{5} \mathrm{Na}\left(\mathrm{M}+\mathrm{Na}^{+}\right)$: 534.2944. Found: 534.2949. 


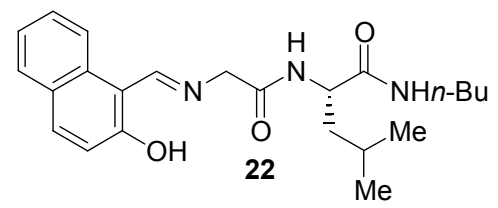

2-Hydroxy-1-napthaldehyde- Gly -L- Leu-NHn-Bu: IR (solid film): 3264 (br), 3069 (w), 2955 (m), 2917 (m), $2873(\mathrm{w}), 1646(\mathrm{~s}), 1540(\mathrm{~m}), 1375$ (m), $1237(\mathrm{~m}), 1193$ (m), $841(\mathrm{w}), 740(\mathrm{w}), 715(\mathrm{w}) \mathrm{cm}^{-1}$. ${ }^{1} \mathrm{H}$ NMR $(400 \mathrm{MHz}$, $\left.\mathrm{CDCl}_{3}\right): \delta 9.10(1 \mathrm{H}, \mathrm{s}, \mathbf{H C}=\mathrm{N}), 8.00(1 \mathrm{H}, \mathrm{d}, J=8.4 \mathrm{~Hz}$, ArH), $7.79(1 \mathrm{H}, \mathrm{d}, J=9.2 \mathrm{~Hz}, \operatorname{ArH}), 7.72(1 \mathrm{H}, \mathrm{d}, J=7.2 \mathrm{~Hz}, \operatorname{ArH}), 7.50(1 \mathrm{H}, \mathrm{dd}, J=$ 7.2, 7.2 Hz, ArH), 7.33 (1H, dd, $J=7.2,7.2 \mathrm{~Hz}, \operatorname{ArH}), 7.09(1 \mathrm{H}, \mathrm{d}, J=9.6 \mathrm{~Hz}, \operatorname{ArH})$, $6.63(1 \mathrm{H}, \mathrm{d}, J=8.0 \mathrm{~Hz}, \mathrm{NH}), 6.15(1 \mathrm{H}$, br s, NH), 4.50-4.35 (3H, m, NCHCO), 3.32$3.16\left(2 \mathrm{H}, \mathrm{m}, \mathrm{NCH}_{2}\right), 1.74-1.44(5 \mathrm{H}$, br m, aliphatic $\mathrm{CH}), 1.32(2 \mathrm{H}$, ddddd, $J=7.6,7.6$, 7.6, 7.6, 7.6 Hz, aliphatic $\mathrm{CH}), 0.93\left(6 \mathrm{H}, \mathrm{d}, J=6.0 \mathrm{~Hz}, \mathrm{CH}\left(\mathrm{CH}_{3}\right)_{2}\right), 0.90(3 \mathrm{H}, \mathrm{dd}, J=7.6$, $\left.7.6 \mathrm{~Hz}, \mathrm{CH}_{3}\right) .{ }^{13} \mathrm{C} \mathrm{NMR}\left(100 \mathrm{MHz}, \mathrm{CDCl}_{3}\right): \delta 171.4,168.7,167.3,163.7,136.3,133.0$, $129.4,128.2,127.5,123.7,121.3,119.1,108.5,60.6,52.1,41.2,39.5,31.7,25.0,22.9$, 22.5, 20.2, 13.8. HRMS Calcd for $\mathrm{C}_{23} \mathrm{H}_{31} \mathrm{~N}_{3} \mathrm{O}_{3} \mathrm{Na}\left(\mathrm{M}+\mathrm{Na}^{+}\right)$: 420.2263. Found: 420.2263 .

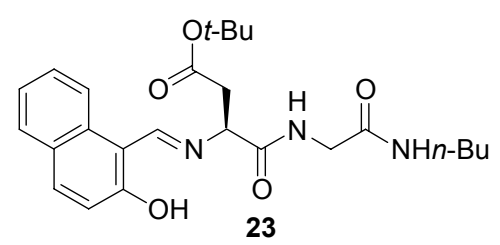

2-Hydroxy-1-napthaldehyde- L-Asp (Ot-Bu)- Gly-NHnBu: IR (solid film): 3295 (br), 3056 (w), 2968 (m), 2943 (m), $2880(\mathrm{w}), 1728(\mathrm{~m}), 1671(\mathrm{~s}), 1650(\mathrm{~s}), 1552(\mathrm{~m})$, 1369 (m), 1155 (m), $909(\mathrm{w}), 847$ (w), $740(\mathrm{~m}) \mathrm{cm}^{-1} \cdot{ }^{1} \mathrm{H}$ NMR (400 MHz, $\left.\mathrm{CDCl}_{3}\right): \delta 9.27(1 \mathrm{H}, \mathrm{s}, \mathbf{H C}=\mathrm{N}), 8.05$ $(1 \mathrm{H}, \mathrm{d}, J=8.4 \mathrm{~Hz}, \operatorname{ArH}), 7.83(1 \mathrm{H}, \mathrm{d}, J=9.2 \mathrm{~Hz}, \operatorname{ArH})$, $7.75(1 \mathrm{H}, \mathrm{d}, J=8.0 \mathrm{~Hz}, \operatorname{ArH}), 7.53(1 \mathrm{H}, \mathrm{ddd}, J=6.8,6.8,1.2 \mathrm{~Hz}, \operatorname{ArH}), 7.36$ (1H, ddd, $J$ $=7.2,7.2,0.8 \mathrm{~Hz}, \operatorname{ArH}), 7.13(1 \mathrm{H}, \mathrm{d}, J=7.2 \mathrm{~Hz}, \operatorname{ArH}), 6.93(1 \mathrm{H}, \mathrm{br} \mathrm{s}, \mathrm{NH}), 6.82(1 \mathrm{H}, \mathrm{br}$ s, NH), $4.33(1 \mathrm{H}, \mathrm{dd}, J=4.8,4.8 \mathrm{~Hz}, \mathrm{NCHCO}), 4.21(1 \mathrm{H}, \mathrm{dd}, J=16.4,7.2 \mathrm{~Hz}$, NCHHCO), $3.71(1 \mathrm{H}, \mathrm{dd}, J=16.4,4.8 \mathrm{~Hz}, \mathrm{NCHHCO}), 3.33-3.16\left(3 \mathrm{H}, \mathrm{m}, \mathrm{NCH}_{2}\right.$ and CHCHHCO), 2.89 (1H, dd, $J=16.4,4.8 \mathrm{~Hz}, \mathrm{CHCHHCO}), 1.55-1.46$ (2H, br m, aliphatic CH), $1.49(9 \mathrm{H}, \mathrm{s}, t-\mathbf{B u}), 1.39-1.25(2 \mathrm{H}, \mathrm{m}$, aliphatic CH), $0.90(3 \mathrm{H}, \mathrm{dd}, J=7.2,7.2 \mathrm{~Hz}$, $\left.\mathrm{CH}_{3}\right) .{ }^{13} \mathrm{C}$ NMR $\left(100 \mathrm{MHz}, \mathrm{CDCl}_{3}\right): \delta 171.0,170.4,168.5,164.8,164.8,136.0,132.8$, $129.4,128.3,127.8,123.9,120.3,119.2,108.7,82.3,68.8,43.5,40.0,39.6,31.6,28.2$, 20.2, 13.9. HRMS Calcd for $\mathrm{C}_{25} \mathrm{H}_{33} \mathrm{~N}_{3} \mathrm{O}_{5} \mathrm{H}\left(\mathrm{M}+\mathrm{H}^{+}\right)$: 456.2498. Found: 456. 2496.

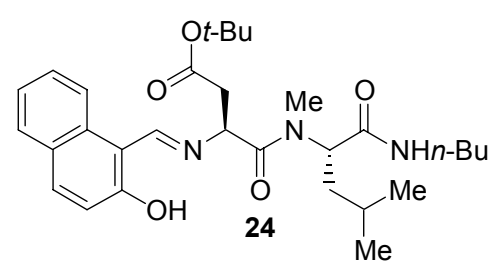

2-Hydroxy-1-napthaldehyde- L-Asp (Ot-Bu)-LLeu(NMe)-NHn-Bu: IR (solid film): 3396 (br), 3320 (m), $3056(\mathrm{w}), 2968$ (s), 2930 (s), $2886(\mathrm{~m}), 1728(\mathrm{~m})$, $1665(\mathrm{~s}), 1646(\mathrm{~s}), 1545(\mathrm{~m}), 1483(\mathrm{~m}), 1369(\mathrm{~s}), 1162(\mathrm{~s})$, $841(\mathrm{~m}), 759(\mathrm{~m}), 734(\mathrm{~m}) \mathrm{cm}^{-1} .{ }^{1} \mathrm{H}$ NMR $(400 \mathrm{MHz}$, $\left.\mathrm{CDCl}_{3}\right): \delta 9.20(1 \mathrm{H}, \mathrm{s}, \mathbf{H C}=\mathrm{N}), 7.96(1 \mathrm{H}, \mathrm{d}, J=8.4 \mathrm{~Hz}$, $\operatorname{ArH}), 7.79(1 \mathrm{H}, \mathrm{d}, J=8.8 \mathrm{~Hz}, \operatorname{ArH}), 7.71(1 \mathrm{H}, \mathrm{d}, J=8.0 \mathrm{~Hz}, \operatorname{ArH}), 7.48(1 \mathrm{H}, \mathrm{ddd}, J=$ 6.8, 6.8, $1.2 \mathrm{~Hz}, \operatorname{ArH}), 7.32(1 \mathrm{H}, \mathrm{ddd}, J=8.0,8.0,0.8 \mathrm{~Hz}, \operatorname{ArH}), 7.08(1 \mathrm{H}, \mathrm{d}, J=8.8 \mathrm{~Hz}$, ArH), 6.50 ( $1 \mathrm{H}, \mathrm{dd}, J=5.6,5.6 \mathrm{~Hz}, \mathrm{NH}), 5.32(1 \mathrm{H}, \mathrm{dd}, J=10.4,4.8 \mathrm{~Hz}, \mathrm{NCHCO}), 5.07$ $(1 \mathrm{H}, \mathrm{dd}, J=9.6,4.0 \mathrm{~Hz}, \mathrm{NCHCO}), 3.35-3.15\left(2 \mathrm{H}, \mathrm{m}, \mathrm{NCH}_{2}\right), 3.29$ (1H, dd, $J=16.8,9.6$ $\mathrm{Hz}, \mathrm{CHCHHCO}), 3.01$ ( $\left.3 \mathrm{H}, \mathrm{s}, \mathrm{NCH}_{3}\right), 2.80(1 \mathrm{H}, \mathrm{dd}, J=16.8,4.8 \mathrm{~Hz}, \mathrm{CHCHHCO})$, 1.90-1.83 $(1 \mathrm{H}, \mathrm{m}$, aliphatic $\mathrm{CH}), 1.68-1.60(1 \mathrm{H}, \mathrm{m}$, aliphatic $\mathrm{CH}), 1.56-1.47(2 \mathrm{H}, \mathrm{m}$, aliphatic CH), $1.45(9 \mathrm{H}, \mathrm{s}, t-\mathbf{B u}), 1.34-1.25(3 \mathrm{H}, \mathrm{m}$, aliphatic $\mathrm{CH}), 0.92(3 \mathrm{H}, \mathrm{dd}, J=7.2$, $\left.7.2 \mathrm{~Hz}, \mathrm{CH}_{3}\right), 0.91\left(6 \mathrm{H}, \mathrm{d}, J=6.8 \mathrm{~Hz}, \mathrm{CH}\left(\mathrm{CH}_{3}\right)_{2}\right), 0.85\left(3 \mathrm{H}, \mathrm{d}, J=6.8 \mathrm{~Hz}, \mathrm{CH}\left(\mathrm{CH}_{3}\right)_{2}\right)_{\text {, }}$. ${ }^{13} \mathrm{C}$ NMR $\left(100 \mathrm{MHz} \mathrm{CDCl}_{3}\right): \delta 171.4,170.8,170.2,167.0,161.0,135.9,133.0,129.4$, 
$128.0,127.5,123.6,121.3,119.0,108.5,82.1,60.1,55.3,39.6,36.0,31.8,30.7,28.2$, 25.1, 23.5, 21.7, 20.3, 13.9. HRMS Calcd for $\mathrm{C}_{30} \mathrm{H}_{43} \mathrm{~N}_{3} \mathrm{O}_{5} \mathrm{H}\left(\mathrm{M}+\mathrm{H}^{+}\right)$: 526.3281. Found: 526.3282 .

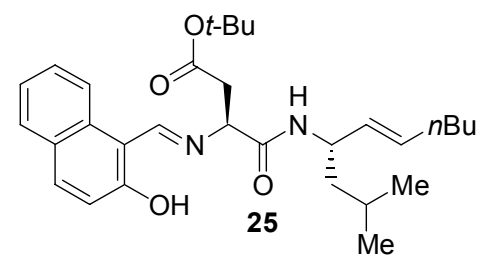

2-Hydroxy-1-napthaldehyde- L-Asp (Ot-Bu)-NH(4-(S)2-methyl-5-decene): IR (solid film): 3283 (br), 3069 (w), 2962 (m), 2924 (m), 2880 (w), 1741 (m), 1653 (m), 1640 (s), $1552(\mathrm{~m}), 1376(\mathrm{~m}), 1161(\mathrm{~m}), 972(\mathrm{w}), 853(\mathrm{~m}), 752$ (m) $\mathrm{cm}^{-1} .{ }^{1} \mathrm{H}$ NMR $\left(400 \mathrm{MHz}, \mathrm{CDCl}_{3}\right): \delta 9.26(1 \mathrm{H}, \mathrm{s}$, $\mathbf{H C}=\mathrm{N}), 8.08(1 \mathrm{H}, \mathrm{d}, J=7.6 \mathrm{~Hz}, \operatorname{ArH}), 7.84(1 \mathrm{H}, \mathrm{d}, J=$ $9.2 \mathrm{~Hz}, \operatorname{ArH}), 7.75(1 \mathrm{H}, \mathrm{d}, J=8.0 \mathrm{~Hz}, \operatorname{ArH}), 7.54(1 \mathrm{H}, \mathrm{ddd}, J=7.2,7.2,1.2 \mathrm{~Hz}, \operatorname{ArH})$, $7.36(1 \mathrm{H}, \mathrm{ddd}, J=7.2,7.2,1.2 \mathrm{~Hz}, \operatorname{ArH}), 7.14(1 \mathrm{H}, \mathrm{d}, J=9.2 \mathrm{~Hz}, \operatorname{ArH}), 5.94(1 \mathrm{H}, \mathrm{d}, J=$ $7.6 \mathrm{~Hz}, \mathrm{NH}), 5.55\left(1 \mathrm{H}, \mathrm{ddd}, J=15.2,6.8,6.8 \mathrm{~Hz}, \mathrm{CH}=\mathrm{CHCH}_{2}\right), 5.24(1 \mathrm{H}, \mathrm{dd}, J=15.2$, $\left.6.8 \mathrm{~Hz}, \mathrm{CH}=\mathrm{CHCH}_{2}\right), 4.52-4.39(2 \mathrm{H}, \mathrm{m}, \mathrm{NCHCO}$ and $\mathrm{NCHCH}=\mathrm{CH}), 3.17(1 \mathrm{H}, \mathrm{dd}, J=$ 16.4, 3.6 Hz, CHCHHCO), 2.79 (1H, dd, $J=16.4,9.6 \mathrm{~Hz}, \mathrm{CHCHHCO}), 1.93$ (2H, ddd, $J$ $\left.=6.8,6.8,6.8 \mathrm{~Hz}, \mathrm{CHCH}_{2}\right), 1.65-1.57(1 \mathrm{H}, \mathrm{m}$, aliphatic $\mathrm{CH}), 1.45-1.16(6 \mathrm{H}, \mathrm{m}$, aliphatic $\mathrm{CH}), 1.35$ (9H, s, $t$-Bu), $0.92\left(6 \mathrm{H}, \mathrm{d}, J=6.8 \mathrm{~Hz}, \mathrm{CH}\left(\mathrm{CH}_{3}\right)_{2}\right), 0.80(3 \mathrm{H}, \mathrm{dd}, J=7.2,7.2$ $\left.\mathrm{Hz}, \mathrm{CH}_{3}\right) .{ }^{13} \mathrm{C}$ NMR $\left(100 \mathrm{MHz}, \mathrm{CDCl}_{3}\right): \delta 170.0,169.2,165.4,164.3,135.9,132.9$, $132.3,129.8,129.4,128.3,127.8,123.8,120.5,119.4,108.7,81.6,69.0,49.8,44.6,40.1$, 32.0, 31.3, 28.2, 25.0, 22.8, 22.7, 22.3, 14.0. HRMS Calcd for $\mathrm{C}_{30} \mathrm{H}_{42} \mathrm{~N}_{2} \mathrm{O}_{4} \mathrm{H}\left(\mathrm{M}+\mathrm{H}^{+}\right)$: 495.3223. Found: 495.3221 .

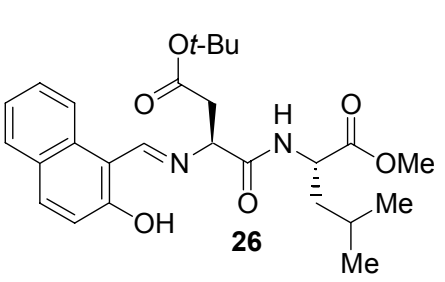

2-Hydroxy-1-napthaldehyde- L-Asp (Ot-Bu)-L-Leu-OMe: IR (solid film): 3301 (br), 3056 (w), 2967 (m), 2924 (m), $2880(\mathrm{w}), 1741(\mathrm{~m}), 1678(\mathrm{~m}), 1640(\mathrm{~s}), 1552(\mathrm{~m}), 1376(\mathrm{~m})$, $1161(\mathrm{~m}), 916(\mathrm{w}), 841(\mathrm{~m}), 746(\mathrm{~m}) \mathrm{cm}^{-1}$. ${ }^{1} \mathrm{H}$ NMR (400 $\left.\mathrm{MHz}, \mathrm{CDCl}_{3}\right): \delta 9.29(1 \mathrm{H}, \mathrm{s}, \mathrm{HC}=\mathrm{N}), 8.07(1 \mathrm{H}, \mathrm{d}, J=8.4$ $\mathrm{Hz}, \operatorname{ArH}), 7.82(1 \mathrm{H}, \mathrm{d}, J=8.4 \mathrm{~Hz}, \operatorname{ArH}), 7.74(1 \mathrm{H}, \mathrm{d}, J=$ $8.0 \mathrm{~Hz}, \operatorname{ArH}), 7.53(1 \mathrm{H}, \mathrm{ddd}, J=7.2,7.2,1.2 \mathrm{~Hz}, \operatorname{ArH}), 7.35(1 \mathrm{H}, \mathrm{dd}, J=7.2,7.2 \mathrm{~Hz}$, $\operatorname{ArH}), 7.14(1 \mathrm{H}, \mathrm{d}, J=8.4 \mathrm{~Hz}, \operatorname{ArH}), 6.54(1 \mathrm{H}, \mathrm{d}, J=8.8 \mathrm{~Hz}, \mathrm{NH}), 4.64(1 \mathrm{H}, \mathrm{m}$, $\mathrm{NCHCO}), 4.48(1 \mathrm{H}, \mathrm{dd}, J=8.8,3.6 \mathrm{~Hz}, \mathrm{NCHCO}), 3.68\left(3 \mathrm{H}, \mathrm{s}, \mathrm{OCH}_{3}\right), 3.15(1 \mathrm{H}, \mathrm{dd}, J=$ 16.4, 4.0 Hz, CHCHHCO), 2.82 (1H, dd, $J=16.4,9.2 \mathrm{~Hz}, \mathrm{CHCHHCO}), 1.72-1.56$ (3H, br m, aliphatic CH), $1.36(9 \mathrm{H}, \mathrm{s}, t-\mathbf{B u}), 0.95\left(6 \mathrm{H}, \mathrm{d}, J=6.0 \mathrm{~Hz}, \mathrm{CH}\left(\mathrm{CH}_{3}\right)_{2}\right) .{ }^{13} \mathrm{C} \mathrm{NMR}$ $\left(100 \mathrm{MHz}, \mathrm{CDCl}_{3}\right): \delta 173.1,170.2,169.8,165.3,164.8,135.9,132.9,129.3,128.2$, 127.7, 123.8, 120.4, 119.4, 108.7, 81.7, 68.5, 52.5, 51.1, 41.3, 40.0, 28.1, 25.1, 22.9, 22.1. HRMS Calcd for $\mathrm{C}_{26} \mathrm{H}_{34} \mathrm{~N}_{2} \mathrm{O}_{6} \mathrm{Na}\left(\mathrm{M}+\mathrm{Na}^{+}\right)$: 493.2311. Found: 493.2315 .

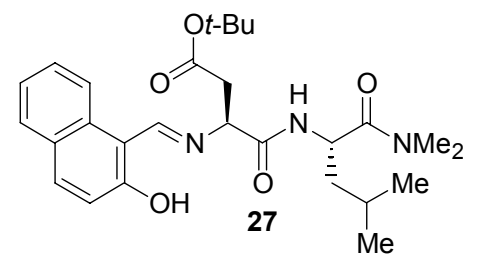

2-Hydroxy-1-napthaldehyde- L-Asp (Ot-Bu)-L-Leu$\mathbf{N M e}_{2}$ : IR (solid film): 3283 (br), 3062 (w), 2968 (m), 2943 (m), $2880(\mathrm{w}), 1734(\mathrm{~m}), 1653(\mathrm{~m}), 1646(\mathrm{~s}), 1552(\mathrm{~m})$, $1420(\mathrm{~m}), 1370(\mathrm{~m}), 1161(\mathrm{~m}), 841(\mathrm{w}), 752(\mathrm{~m}) \mathrm{cm}^{-1} .{ }^{1} \mathrm{H}$ NMR $\left(400 \mathrm{MHz}, \mathrm{CDCl}_{3}\right): \delta 9.26(1 \mathrm{H}, \mathrm{s}, \mathbf{H C}=\mathrm{N}), 8.06(1 \mathrm{H}$, $\mathrm{d}, J=8.8 \mathrm{~Hz}, \operatorname{ArH}), 7.81(1 \mathrm{H}, \mathrm{d}, J=6.8 \mathrm{~Hz}, \operatorname{ArH}), 7.73$ $(1 \mathrm{H}, \mathrm{dd}, J=8.0,1.2 \mathrm{~Hz}, \operatorname{ArH}), 7.51(1 \mathrm{H}, \mathrm{ddd}, J=6.8,6.8,1.2 \mathrm{~Hz}, \operatorname{ArH}), 7.34(1 \mathrm{H}, \mathrm{ddd}$, $J=7.2,7.2,1.2 \mathrm{~Hz}, \operatorname{ArH}), 7.14(1 \mathrm{H}, \mathrm{d}, J=8.8 \mathrm{~Hz}, \operatorname{ArH}), 6.86(1 \mathrm{H}, \mathrm{d}, J=8.0 \mathrm{~Hz}, \mathrm{NH})$, 
$5.02(1 \mathrm{H}, \mathrm{ddd}, J=10.0,10.0,4.0 \mathrm{~Hz}, \mathrm{NCHCO}), 4.45(1 \mathrm{H}, \mathrm{dd}, J=9.6,4.0 \mathrm{~Hz}, \mathrm{NCHCO})$, $3.15(1 \mathrm{H}, \mathrm{dd}, J=16.4,3.6 \mathrm{~Hz}, \mathrm{CHCHHCO}), 3.08\left(3 \mathrm{H}, \mathrm{s}, \mathrm{NCH}_{3}\right), 2.89\left(3 \mathrm{H}, \mathrm{s}, \mathrm{NCH}_{3}\right)$, $2.81(1 \mathrm{H}, \mathrm{dd}, J=16.4,9.6 \mathrm{~Hz}, \mathrm{CHCHHCO}), 1.71-1.41(3 \mathrm{H}$, br m, aliphatic CH), 1.35 $(9 \mathrm{H}, \mathrm{s}, t-\mathbf{B u}), 1.01\left(3 \mathrm{H}, \mathrm{d}, J=6.8 \mathrm{~Hz}, \mathrm{CH}\left(\mathrm{CH}_{3}\right)_{2}\right), 0.93\left(3 \mathrm{H}, \mathrm{d}, J=6.8 \mathrm{~Hz}, \mathrm{CH}\left(\mathrm{CH}_{3}\right)_{2}\right)$. ${ }^{13} \mathrm{C} \mathrm{NMR}\left(100 \mathrm{MHz}, \mathrm{CDCl}_{3}\right): \delta 172.0,169.9,165.5,164.6,164.5,135.7,133.0,129.3$, $128.1,127.7,123.6,120.7,119.5,109.7,81.6,68.6,47.8,42.3,20.1$, 37.1, 35.9, 28.1, 24.9, 23.6, 22.1. HRMS Calcd for $\mathrm{C}_{27} \mathrm{H}_{3} \mathrm{~N}_{3} \mathrm{O}_{5} \mathrm{H}\left(\mathrm{M}+\mathrm{H}^{+}\right)$: 484.2806. Found: 484.2811.

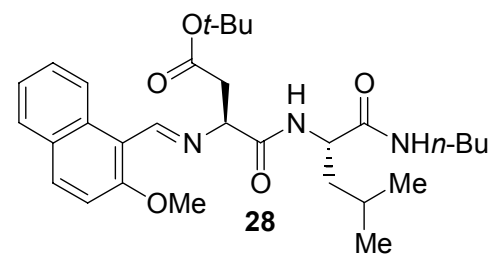

2-Methoxy-1-napthaldehyde- L-Asp (OtBu)-L-LeuNHnBu: IR (solid film): 3314 (br), 3068 (m), 2968 (m), 2930 (m), 2867 (m), 1722 (s), 1646 (s), 1539 (s), 1457 (s), 1357 (s), 1237 (s), 1155 (s), 1080 (s), 916 (m), $822(\mathrm{~m})$, $727(\mathrm{~m}) \mathrm{cm}^{-1}$. ${ }^{1} \mathrm{H}$ NMR $\left(400 \mathrm{MHz}, \mathrm{CDCl}_{3}\right): \delta 9.07(1 \mathrm{H}, \mathrm{s}$, $\mathbf{H C}=\mathrm{N}), 8.91(1 \mathrm{H}, \mathrm{d}, J=8.8 \mathrm{~Hz}, \operatorname{ArH}), 7.90(1 \mathrm{H}, \mathrm{d}, J=$ $9.2 \mathrm{~Hz}, \operatorname{ArH}), 7.76(1 \mathrm{H}, \mathrm{d}, J=8.4 \mathrm{~Hz}, \operatorname{ArH}), 7.53-7.48(1 \mathrm{H}, \mathrm{m}, \operatorname{ArH}), 7.38-7.34(1 \mathrm{H}, \mathrm{m}$, $\operatorname{ArH}), 7.26(1 \mathrm{H}, \mathrm{d}, J=8.8 \mathrm{~Hz}, \operatorname{ArH}), 7.16(1 \mathrm{H}, \mathrm{d}, J=8.0 \mathrm{~Hz}, \mathrm{NH}), 6.51(1 \mathrm{H}, \mathrm{br} \mathrm{s}, \mathrm{NH})$, 4.48-4.43 (1H, m, NCHCO), $4.36(1 \mathrm{H}, \mathrm{dd}, J=10.0,3.2 \mathrm{~Hz}, \mathrm{NCHCO}), 3.98(3 \mathrm{H}, \mathrm{s}$, $\left.\mathrm{OCH}_{3}\right), 3.23-3.12\left(2 \mathrm{H}, \mathrm{m}, \mathrm{NCH}_{2}\right), 3.08(1 \mathrm{H}, \mathrm{dd}, J=16.0,3.2 \mathrm{~Hz}, \mathrm{CHCHHCO}), 2.78$ $(1 \mathrm{H}, \mathrm{dd}, J=16.0,10.0 \mathrm{~Hz}, \mathrm{CHCHHCO}), 1.77-1.39(7 \mathrm{H}, \mathrm{m}$, aliphatic CH), $1.36(9 \mathrm{H}, \mathrm{s}, t-$ Bu ), 1.33-1.20 (2H, m, aliphatic CH), $0.93\left(3 \mathrm{H}, \mathrm{d}, J=7.2, \mathrm{~Hz}, \mathrm{CH}\left(\mathrm{CH}_{3}\right)_{2}\right), 0.92(3 \mathrm{H}, \mathrm{d}, J$ $\left.=7.2, \mathrm{~Hz}, \mathrm{CH}\left(\mathrm{CH}_{3}\right)_{2}\right), 0.84\left(3 \mathrm{H}, \mathrm{d}, J=7.2 \mathrm{~Hz}, \mathrm{CH}_{3}\right) .{ }^{13} \mathrm{C} \mathrm{NMR}\left(100 \mathrm{MHz}, \mathrm{CDCl}_{3}\right)$ : $\delta 172.5,171.5,170.1,163.2,158.3,133.5,132.2,129.0,128.4,128.2,125.0,124.3$, $116.5,112.7,81.2,71.0,56.5,51.8,40.3,39.2,31.5,28.1,25.0,23.1,22.2,20.1,13.8$. HRMS Calcd for $\mathrm{C}_{30} \mathrm{H}_{43} \mathrm{~N}_{3} \mathrm{O}_{5} \mathrm{Na}\left(\mathrm{M}+\mathrm{Na}^{+}\right)$: 548.3108. Found: 548.3100 .

Synthesis of $(\boldsymbol{R})-(+)-4-(2-n i t r o p h e n y l)-1-($ diethylphophoryloxy)-2-hexene: $(R)-(+)-3-$ (2-Nitro-phenyl)-1-pentene (5e) $\left(0.11 \mathrm{~g}, 0.55 \mathrm{mmol}\right.$ includes $\mathrm{S}_{\mathrm{N}} 2$ product) and cis-2butene-1,4-diacetate $\left(9.5 \times 10^{-2} \mathrm{~g}, 0.55 \mathrm{mmol}\right)$ are dissolved in $3.5 \mathrm{~mL} \mathrm{CH}_{2} \mathrm{Cl}_{2}$ and $\mathrm{Ru}-$ catalyst $\left(3.5 \mathrm{mg}, 5.5 \times 10^{-2} \mathrm{mmol}\right)$ is added in one portion under a nitrogen atmosphere. After $24 \mathrm{~h}$, the reaction is concentrated in vacuo. The resulting brown solution is purified by silica gel column chromatography $\left(5: 1\right.$ hexanes/EtOAc, $\mathrm{R}_{f}$ product $\left.=0.17\right)$ to yield a colorless oil $\left(5.1 \times 10^{-2} \mathrm{~g}, 0.19 \mathrm{mmol}, 73 \%\right.$ based on $58 \%$ conversion). $(R)-(+)-1-$ Acetoxy-4-(2-nitrophenyl)-2-hexene $\left(8.0 \times 10^{-2} \mathrm{~g}, 0.30 \mathrm{mmol}\right)$ is dissolved in $1.5 \mathrm{~mL}$ $\mathrm{CH}_{2} \mathrm{Cl}_{2}$ followed by the addition of $\mathrm{MeOH}(0.75 \mathrm{~mL})$ and $\mathrm{K}_{2} \mathrm{CO}_{3}(0.17 \mathrm{~g}, 1.2 \mathrm{mmol})$. After $2 \mathrm{~h}$, TLC analysis shows complete conversion to product (1:1 hexanes/EtOAc; $\mathrm{R}_{f}$ starting material $=0.69, \mathrm{R}_{f}$ product $\left.=0.31\right)$. The reaction is quenched by the addition of 5 $\mathrm{mL} 10 \%$ aqueous $\mathrm{HCl}$, and extracted with EtOAc $(3 \times 3.0 \mathrm{~mL})$. The combined organic layers are dried over anhydrous $\mathrm{MgSO}_{4}$, filtered, and concentrated to yield a yellow oil which is purified by silica gel column chromatography $\left(5.8 \times 10^{-2} \mathrm{~g}, 0.26 \mathrm{mmol}, 86 \%\right)$. $(R)-(+)-4-(2-N i t r o p h e n y l) h e x-2-e n-1-o l$ is converted to the derived phosphate by a previously reported procedure. ${ }^{3}$

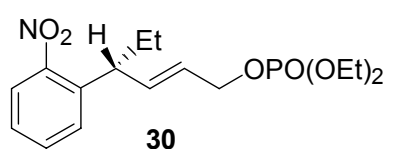

30
(R)-(+)-4-(2-Nitrophenyl)-1-(diethylphophoryloxy)-2hexene. IR (neat): 3465 (w), 3069 (m), 2962 (s), 2930 (s), 2873 (s), 1665 (w), 1615 (m), 1539 (s), $1464(\mathrm{~m}), 1357$ (s), 
1281 (s), 1162 (s), 1017 (s), 859 (s), 753 (s) cm ${ }^{-1}$. ${ }^{1} \mathrm{H}$ NMR (400 MHz, $\mathrm{CDCl}_{3}$ ): $\delta 7.72$ $(1 \mathrm{H}, \mathrm{dd}, J=8.0,1.2 \mathrm{~Hz}, \operatorname{ArH}), 7.54(1 \mathrm{H}, \mathrm{dd}, J=8.0,8.0,1.6 \mathrm{~Hz}, \operatorname{ArH}), 7.39(1 \mathrm{H}, \mathrm{dd}, J=$ 8.0, $1.2 \mathrm{~Hz}, \operatorname{ArH}), 7.33$ (1H, ddd, $J=8.0,8.0,1.2 \mathrm{~Hz}, \operatorname{ArH}), 5.91(1 \mathrm{H}, \mathrm{dd}, J=15.6,8.8$ $\left.\mathrm{Hz}, \mathrm{CH}=\mathrm{CHCH}_{2} \mathrm{O}\right), 5.64\left(1 \mathrm{H}\right.$, dddd, $\left.J=15.6,6.0,6.0,1.2 \mathrm{~Hz}, \mathrm{CH}=\mathrm{CHCH}_{2} \mathrm{O}\right), 4.52-4.48$ $\left(2 \mathrm{H}, \mathrm{m}, \mathrm{CH}=\mathrm{CHCH}_{2} \mathrm{O}\right), 4.13-4.03\left(4 \mathrm{H}, \mathrm{m}, \mathrm{PO}_{2}\left(\mathrm{OCH}_{2} \mathrm{CH}_{3}\right)\right), 3.80(1 \mathrm{H}, \mathrm{dd}, J=7.2,7.2$ $\left.\mathrm{Hz}, \mathrm{CHCH}_{2} \mathrm{CH}_{3}\right), 1.83-1.74\left(2 \mathrm{H}, \mathrm{m}, \mathrm{CHCH}_{2} \mathrm{CH}_{3}\right), 1.30(6 \mathrm{H}$, dddd, $J=10.4,7.2,7.2,0.8$ $\left.\mathrm{Hz}, \mathrm{PO}_{2}\left(\mathrm{OCH}_{2} \mathrm{CH}_{3}\right)\right), 0.87\left(3 \mathrm{H}, \mathrm{dd}, J=7.2,7.2 \mathrm{~Hz}, \mathrm{CHCH}_{2} \mathrm{CH}_{3}\right) .{ }^{13} \mathrm{C} \mathrm{NMR}(100 \mathrm{MHz}$, $\left.\mathrm{CDCl}_{3}\right): \delta 150.4,138.1,136.7,132.7,129.1,127.2,126.1(\mathrm{~d}, J=6.4 \mathrm{~Hz}), 124.2,67.6(\mathrm{~d}$, $J=5.3 \mathrm{~Hz}), 63.9(\mathrm{~d}, J=5.0 \mathrm{~Hz}), 63.8(\mathrm{~d}, J=5.5 \mathrm{~Hz}), 43.7,28.6,16.3(\mathrm{~d}, J=6.8 \mathrm{~Hz})$, 12.1. HRMS Calcd for $\mathrm{C}_{16} \mathrm{H}_{24} \mathrm{NO}_{6} \mathrm{P}: 357.1341$. Found: 357.1344 . $[\alpha]_{\mathrm{D}}{ }^{20}+11.1^{\circ}(c=$ $\left.2.53, \mathrm{CHCl}_{3}\right)$.

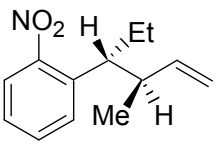

31

(3R,4R)-(+)-4-(2-Nitrophenyl)-3-methylhexene. IR (neat): 3081 (m), 2962 (s), 2937 (m), $2880(\mathrm{~m}), 1532$ (s), $1451(\mathrm{~m}), 1369$ (s), 999 (m), 922 (m), $859(\mathrm{~m}), 803(\mathrm{~m}), 746(\mathrm{~m}) \mathrm{cm}^{-1}$. ${ }^{1} \mathrm{H}$ NMR (400 MHz, $\left.\mathrm{CDCl}_{3}\right): \delta 7.63$ $(1 \mathrm{H}, \mathrm{dd}, J=7.6,1.6 \mathrm{~Hz}, \operatorname{ArH}), 7.55-7.51(1 \mathrm{H}, \mathrm{m}, \operatorname{ArH}), 7.38(1 \mathrm{H}, \mathrm{dd}, J=$ 7.6, 1.6 Hz, ArH), 7.31 (1H, ddd, $J=7.6,7.6,1.2 \mathrm{~Hz}, \operatorname{ArH}), 5.71$ (1H, ddd, $J=17.6,9.6$, $\left.8.8 \mathrm{~Hz}, \mathrm{CHCH}=\mathrm{CH}_{2}\right), 5.05-5.00\left(2 \mathrm{H}, \mathrm{m}, \mathrm{CHCH}=\mathrm{CH}_{2}\right), 2.86(1 \mathrm{H}$, ddd, $J=10.8,9.2,3.6$ $\left.\mathrm{Hz}, \mathrm{CHCH}_{2} \mathrm{CH}_{3}\right), 2.40-2.30\left(1 \mathrm{H}, \mathrm{m}, \mathrm{CHCH}=\mathrm{CH}_{2}\right), 1.92(1 \mathrm{H}$, ddd, $J=14.0,7.6,3.6 \mathrm{~Hz}$, $\left.\mathrm{CHCHHCH}_{3}\right), 1.47\left(1 \mathrm{H}, \mathrm{ddd}, J=14.0,10.8,7.2 \mathrm{~Hz}, \mathrm{CHCHHCH}_{3}\right), 0.81(3 \mathrm{H}, \mathrm{d}, J=6.8$ $\left.\mathrm{Hz}, \mathrm{CHCH}_{3}\right), 0.68\left(3 \mathrm{H}, \mathrm{dd}, J=7.2,7.2 \mathrm{~Hz}, \mathrm{CCH}_{2} \mathrm{CH}_{3}\right) .{ }^{13} \mathrm{C} \mathrm{NMR}\left(100 \mathrm{MHz}, \mathrm{CDCl}_{3}\right): \delta$ $152.5,142.6,138.3,132.0,128.4,126.7,123.4,115.0,46.1,44.8,26.9,19.4,11.9$. HRMS Calcd for $\mathrm{C}_{13} \mathrm{H}_{17} \mathrm{NO}_{2}: 219.1259$. Found: 219.1258. $[\alpha]_{\mathrm{D}}{ }^{20}+44.3^{\circ}(c=0.533$, $\left.\mathrm{CHCl}_{3}\right)$.

\section{Ligand Optimization Studies:}

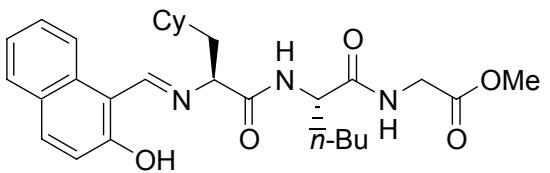

Representative procedure for synthesis of dipeptide ligand on solid support. Gly-Wang resin $(0.10 \mathrm{~g}, 6.0$ $\left.\mathrm{x} 10^{-2} \mathrm{mmol}\right)$ is weighed into a tube and allowed to swell in $2 \mathrm{~mL}$ DMF for $8 \mathrm{~h}$. After this time, DMF is removed by filtration, and Fmoc-L-Nle-OH $\left(8.5 \times 10^{-2} \mathrm{~g}, 0.24 \mathrm{mmol}\right)$ is weighed into the tube with the resin. The amino acid is dissolved in $2 \mathrm{~mL} \mathrm{DMF}$, followed by addition of DIC $\left(4.1 \times 10^{-2} \mathrm{~mL}, 2.7 \times 10^{-2} \mathrm{mmol}\right)$. The tube is then capped and agitated for $3 \mathrm{~h}$. After this time, the excess reagents are filtered off and the resin is washed eight times with $2 \mathrm{~mL}$ of DMF. To deprotect, $2 \mathrm{~mL}$ of a $20 \%$ solution of piperidine in DMF is added to the tubes. The tubes are then recapped and agitated for $30 \mathrm{~min}$. The solution is filtered off and the resin is washed eight times with $2 \mathrm{~mL}$ of DMF. For the second coupling, Fmoc-L-Cha-OH $\left(9.5 \times 10^{-2} \mathrm{~g}, 0.24 \mathrm{mmol}\right), 2 \mathrm{~mL}$ of DMF, and DIC $\left(4.1 \times 10^{-2} \mathrm{~mL}, 2.7 \mathrm{x}\right.$ $10^{-2} \mathrm{mmol}$ ) are added to the tube with the washed resin and the solution is agitated for 3 $\mathrm{h}$. The solution is then filtered, and the resin is washed eight times with $2 \mathrm{~mL}$ of DMF. To deprotect, $2 \mathrm{~mL}$ of a $20 \%$ solution of piperidine in DMF is added to each tube, the tubes recapped and agitated for $30 \mathrm{~min}$. The solution is again filtered and the resin is washed eight times with $2 \mathrm{~mL}$ of DMF. Salicyl aldehyde $\left(4.1 \times 10^{-2} \mathrm{~g}, 0.24 \mathrm{mmol}\right)$ and 2 $\mathrm{mL}$ of $\mathrm{DMF}$ are added to the tube and it is agitated for $48 \mathrm{~h}$. At this time, the solution is filtered and the resin is washed eight times with $2 \mathrm{~mL}$ of DMF, eight times with $2 \mathrm{~mL}$ of 
toluene, and eight times with $2 \mathrm{~mL}$ of diethyl ether. Lastly, the resin is treated with $2 \mathrm{~mL}$ of a 9:1:0.5 MeOH:DMF: $\mathrm{Et}_{3} \mathrm{~N}$ solution and agitated for $12 \mathrm{~h}$. At this time the resin is washed with EtOAc $(8 \times 2 \mathrm{~mL})$. EtOAc is then removed in vacuo and $2 \mathrm{~mL}$ of toluene are added to the yellow solid and then toluene is also removed. The ligand is dried over $\mathrm{P}_{2} \mathrm{O}_{5}$ under vacuum for $24 \mathrm{~h}$ before use.

Ligand Screening Results. ${ }^{7}$ The screening was carried out using the conversion of 6a ( $\left.23 \mathrm{mg}, 8.0 \times 10^{-2} \mathrm{mmol}\right)$ to $7 \mathbf{a}$ as the test reaction. The reactions were carried out using $(\mathrm{CuOTf})_{2} \cdot \mathrm{C}_{6} \mathrm{H}_{6}\left(2.0 \mathrm{mg}, 4.0 \times 10^{-3} \mathrm{mmol}\right)$ and $10 \mathrm{~mol} \%$ ligand $\left(4.1 \mathrm{mg}, 8.0 \times 10^{-3}\right.$ mmol), 3.0 equiv $\mathrm{Et}_{2} \mathrm{Zn}\left(25 \mu \mathrm{L}, 2.4 \times 10^{-1} \mathrm{mmol}\right)$ in $1.0 \mathrm{~mL}$ THF at $-30{ }^{\circ} \mathrm{C}$ for $12 \mathrm{~h}$. The results are depicted for each library screen.

Chart 1. Ligand Screen for $A A_{1}$ Optimization $\left(A A_{2}=N l e\right)$. The library indicated $\mathrm{Asp}(\mathrm{O} t \mathrm{Bu})$ is one optimal $\mathrm{AA}_{1}(68 \%$ ee).

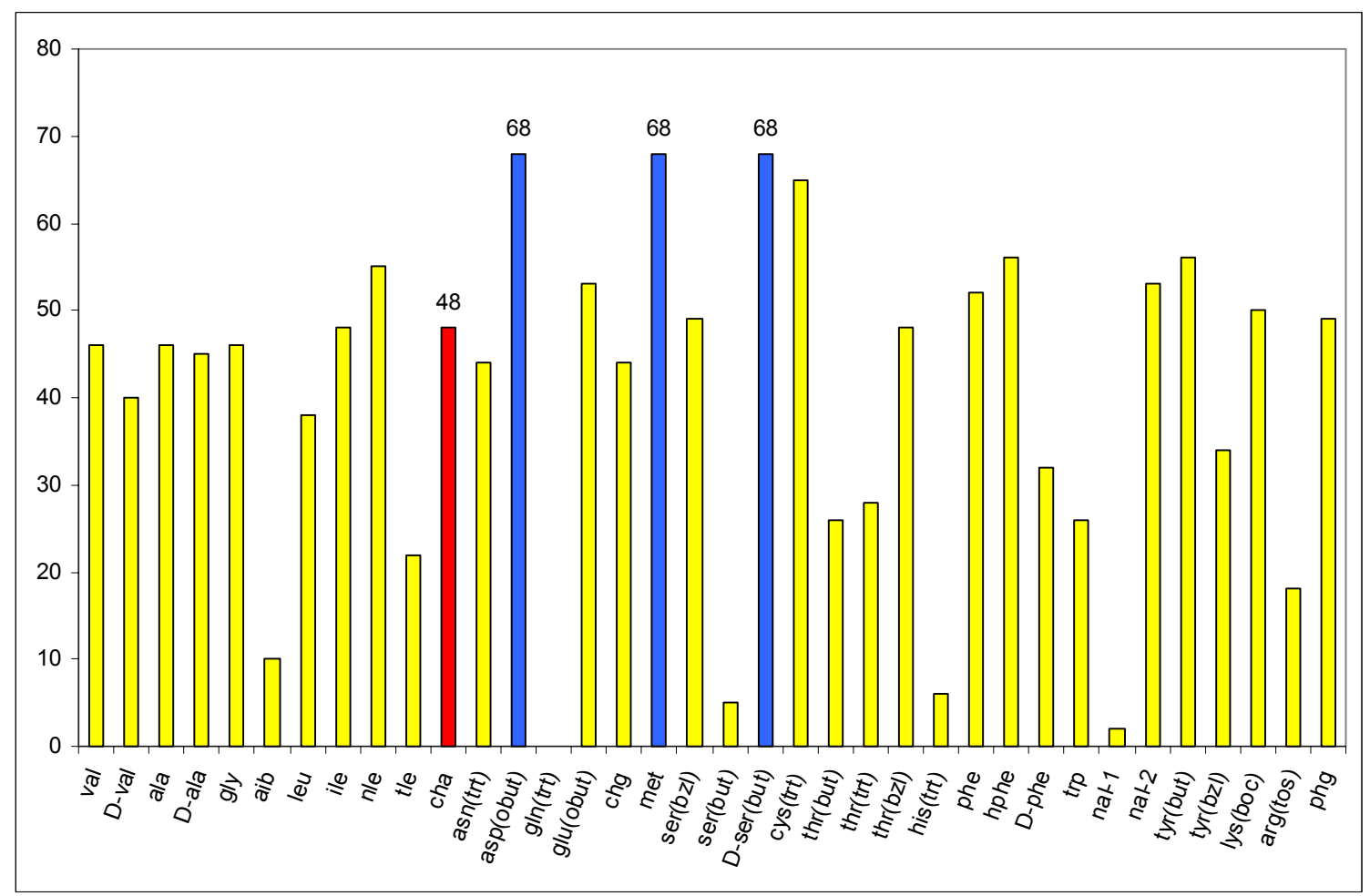

Chart 2. Ligand Screen for $A A_{2}$ Optimization $\left(A A_{1}=A s p(O t B u)\right)$. The library indicated a few amino acids to be optimal $\mathrm{AA}_{2}(83-90 \%$ ee). However, once the ligands were made with the solution phase method, Leu as $A A_{2}$ was optimal ( $92 \%$ ee).

\footnotetext{
${ }^{7}$ B. M. Cole, K. D. Shimizu, C. A. Krueger, J. P. A. Harrity, M. L. Snapper, A. H. Hoveyda, Angew. Chem., Int. Ed. 1996, 35, 1668-1671.
} 


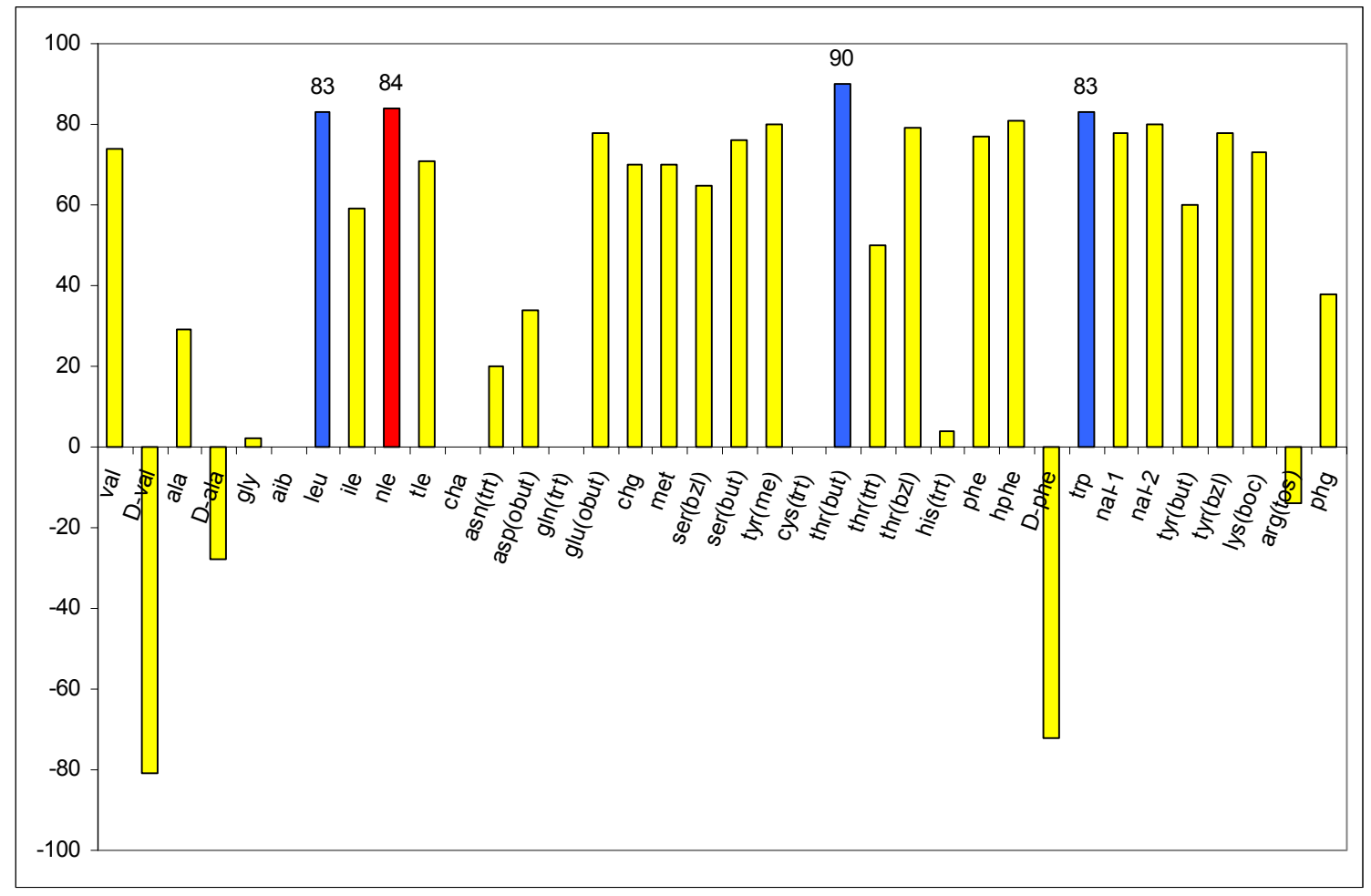

Chart 3. Ligand Screen for Schiff Base Optimization. 19 salicyl aldehydes (Scheme 3) were screened. Aldehydes 2 and $\mathbf{1 0}$ proved to be optimal (92\% ee). 
Kacprzynski \& Hoveyda, Page S24

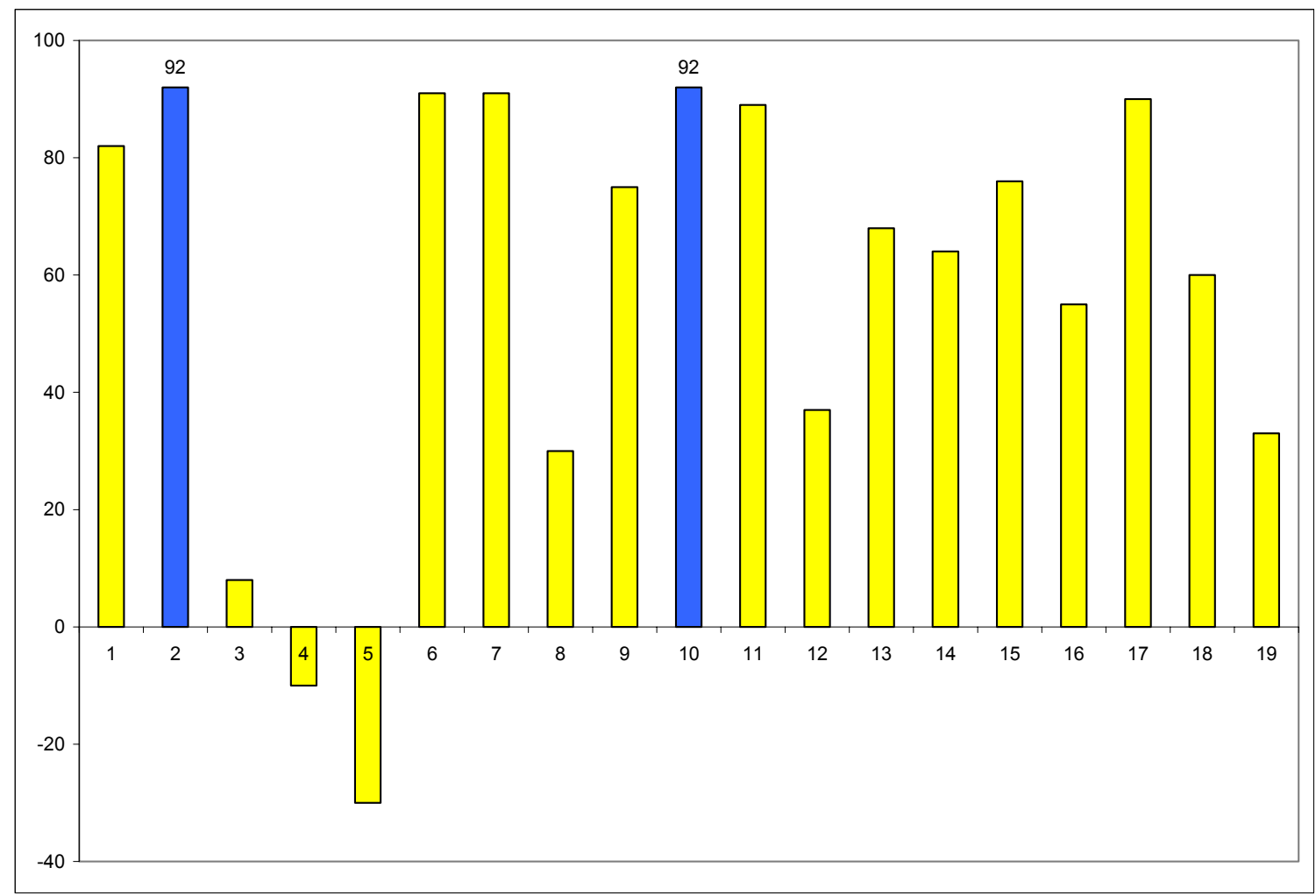

Scheme 4. Aldehydes for Schiff Base Screen

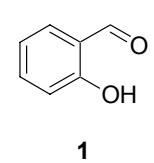<smiles>O=Cc1c(O)ccc2ccccc12</smiles><smiles>O=Cc1cccc(O)c1</smiles><smiles>O=Cc1ccc(O)cc1</smiles><smiles>O=Cc1ccc(O)cc1O</smiles><smiles>O=Cc1cc(O)ccc1O</smiles><smiles>O=Cc1ccccc1C(=O)O</smiles><smiles>O=Cc1cccc(O)c1O</smiles><smiles>COc1ccc(C=O)c(O)c1</smiles><smiles>COc1ccc(O)c(C=O)c1</smiles><smiles>O=Cc1cc([N+](=O)[O-])ccc1O</smiles><smiles>COc1cc([N+](=O)[O-])cc(C=O)c1O</smiles><smiles>COc1cc(Br)cc(C=O)c1O</smiles><smiles>O=Cc1cc(Br)cc([N+](=O)[O-])c1O</smiles>

15

16<smiles>O=Cc1cc(Cl)cc(Cl)c1O</smiles>

17<smiles>O=Cc1cc(Br)cc(Br)c1O</smiles>

18

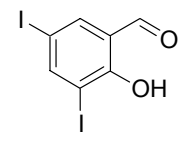

19 